\title{
Poroelastic Finite Element Analysis of a Heterogeneous Articular Cartilage Explant Under Dynamic Compression in ABAQUS
}

\author{
A Thesis Presented to the \\ Faculty of the College of Engineering \\ California Polytechnic State University, San Luis Obispo
}

In Partial Fulfillment of the Requirements for the Degree

Master of Science in Mechanical Engineering

by

Kelsey Kiyo Kam

June 8, 2010 
(C) 2011

Kelsey Kiyo Kam

ALL RIGHTS RESERVED 


\section{Committee Membership}

Title:

Poroelastic Finite Element Analysis of a

Heterogeneous Articular Cartilage Explant Under

Dynamic Compression in ABAQUS

Author:

Kelsey Kiyo Kam

Date Submitted:

June 8, 2011

Committee Chair: Stephen M. Klisch, Professor

Committee Member: Scott Hazelwood, Associate Professor

Committee Member: Peter Schuster, Associate Professor 


\section{Abstract}

\section{Poroelastic Finite Element Analysis of a Heterogeneous Articular Cartilage Explant Under Dynamic Compression in ABAQUS}

\section{Kelsey Kiyo Kam}

A poroelastic finite element model of a heterogeneous articular cartilage disc was created to examine the tissue response to low amplitude ( $\pm 2 \%$ strain), low frequency $(0.1 \mathrm{~Hz})$ dynamic unconfined compression (UCC). A strong correlation has been made between the relative fluid velocity and stimulation of glycosaminoglycan synthesis. A contour plot of the model shows the relative fluid velocity during compression exceeds a trigger value of $0.25 \mu \mathrm{m} / \mathrm{s}$ at the radial periphery. Dynamic UCC biochemical results have also reported a higher glycosaminoglycan content in this region versus that of day 0 specimens. Fluid velocity was also found not to be the dominant physical mechanism that stimulates collagen synthesis; the heterogeneity of the fluid velocity contour plot conflicts with the homogeneous collagen content from the biochemical results. It was also found that a Tresca (shear) stress trigger of $0.07 \mathrm{MPa}$ could provide minor stimulation of glycosaminoglycan synthesis. A feasibility study on modeling a heterogeneous disc was conducted and found convergence issues with the jump in properties from the superficial to middle layers of the disc. It is believed that the superficial layer contains material properties that allow the tissue to absorb much of the compressive strain, which in turn increases pressure and causes convergence issues in ABAQUS. The findings in this thesis may help guide the development of a growth and remodeling routine for articular cartilage. 


\section{Acknowledgements}

This thesis would not have been completed without the help and guidance of many individuals who have provided valuable knowledge and support for this project. I'd like to first and foremost thank my thesis committee. My committee chair Dr. Stephen Klisch and committee member Dr. Scott Hazelwood have provided me with their indispensible knowledge about the mechanics of articular cartilage and modeling in ABAQUS. It has been an honor working with such great individuals who have encouraged me throughout my career at Cal Poly. I would also like to extend my appreciation to my committee member Dr. Peter Schuster, who has challenged me with learning finite element analysis as well as providing help with stubborn convergence issues in ABAQUS.

Much of the analyses could not have been completed without the help from the other students of the Cal Poly Cartilage Biomechanics Group. I would like to most importantly express my gratitude to Mike Stender who has provided his all mighty UMAT for my analyses. Mike has also given me inspiration to work through those stubborn ABAQUS hurdles and kept me sane through those long summer working days. I would also like to thank Kevin Yamauchi for providing the biochemical data to compare my analytical results. He and Matt Griebel have graciously helped me review this thesis.

Finally, I'd like to thank my family and friends who have enveloped me with their love and support through all these years. To my mom and dad who have loved me unconditionally and taught me to always follow my dreams, my grandparents who have supported me through my education, and my brothers who have kept me levelheaded. I'd also like to thank my friends Drew, John, Bryan, and Michael who have been there for me during the stressful times and also pushed me to complete my work.

This project was supported by grants from NSF and NIH. 


\section{Table of Contents}

List of Tables ........................................................................................................... viii

List of Figures.......................................................................................................... ix

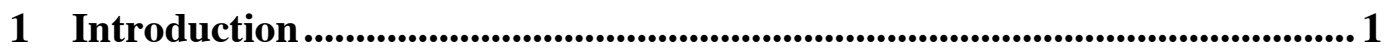

2 Background Review ................................................................................................. 4

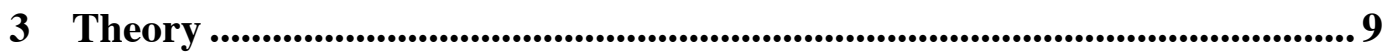

3.1 Kinematics .....................................................................................................

3.2 Biphasic Mixtures........................................................................................... 11

3.3 Constitutive Stress Equations ........................................................................... 14

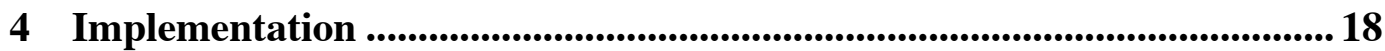

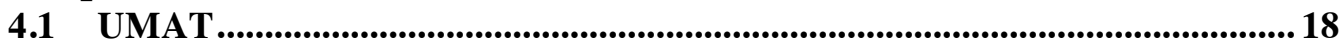

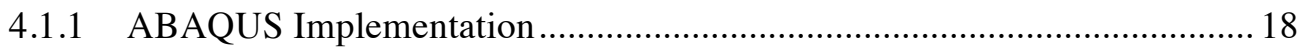

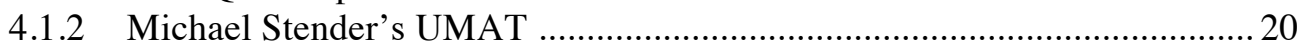

4.2 ABAQUS Modeling .................................................................................... 20

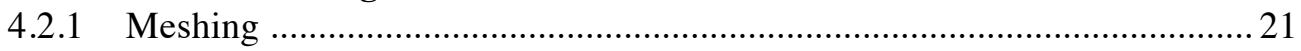

4.2.2 Material Properties …………………………………………………. 22

4.2.3 Node and Element Sets …………………………………………..... 26

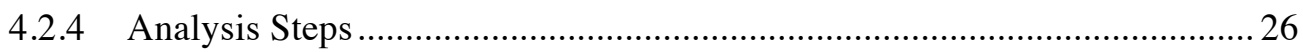

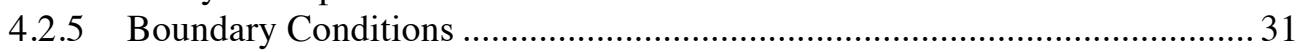

4.3 Keywords Adjustments.....................................................................................33

5 Experimental Results and FEA Parameters for Dynamic UCC ............... 35

5.1 Dynamic UCC Experimental Data ......................................................................35

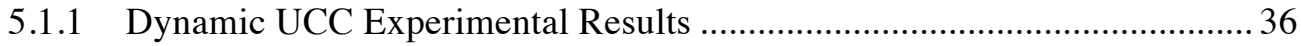

5.2 FEA Material Properties .........................................................................................4 40

5.2.1 Heterogeneous Model ............................................................................ 40

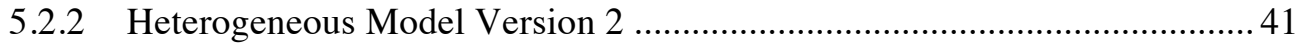

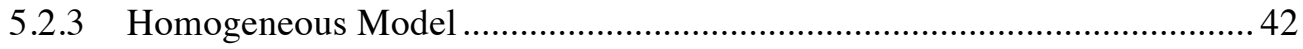

5.3 Physical Mechanisms for Biosynthesis ..................................................................43

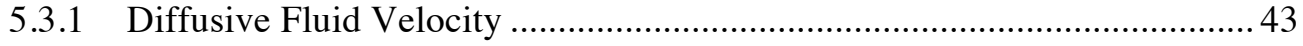

5.3.2 Maximum Shear Stress ……………………………………………..... 44

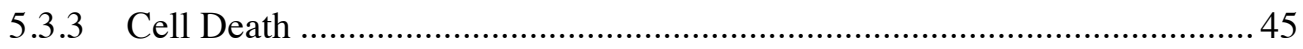

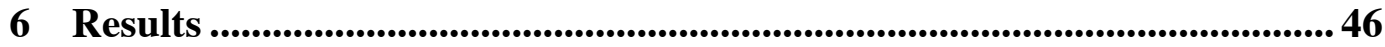

6.1 Diffusive Fluid Velocity Results ......................................................................46

6.2 Tresca Stress Results......................................................................................48

6.3 Radial Strain Results ......................................................................................50

6.4 Cell Death Results ............................................................................................52

6.4.1 Minimum Principal Stress Results ............................................................... 53

6.4.2 Minimum Principal Strain Results..............................................................54

6.4.3 Maximum Principal Stress Results .............................................................. 55

6.4.4 Maximum Principal Strain Results .......................................................... 56

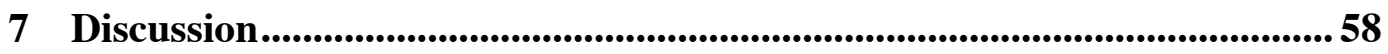

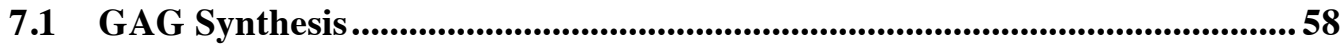

7.2 COL Synthesis .........................................................................................................59 
$7.3 \quad$ Feasibility of Heterogeneity ............................................................................61

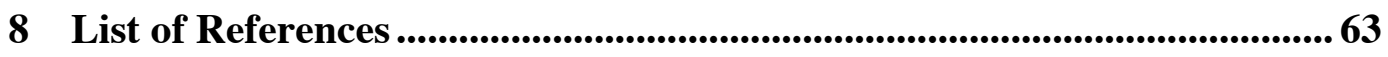

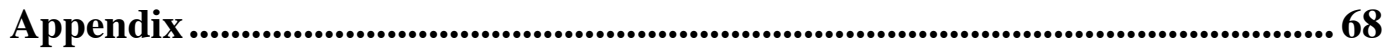

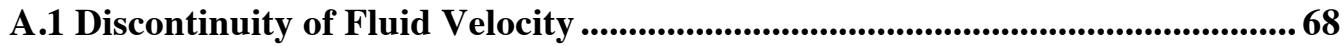

A.2 Suggestions for Future Work .................................................................................. 71

A.3 Previous Failed Attempts at Convergence ............................................................... 72

A.4 Technical Specifications ................................................................................................ 76

A.5 Statistical Data on COL/GAG Concentrations ............................................... 77 


\section{List of Tables}

Table 4.1. Material property variables set in the UMAT for the $S$ and $M$ layers

Table 4.2. Experimental permeability values $\left[\mathrm{m}^{2} / \mathrm{Pa}-\mathrm{s}\right]$ for the $\mathrm{S}$ and $\mathrm{M}$ layers at different CC strains [34].

Table 4.3. The time periods and incrementation sizes chosen for the analysis steps

Table 5.1. Experimental results for D0 specimens from [34] and FSB and S10+D data from [37]. Biochemical data is presented for GAG and COL content as a percentage of wet weight.

Table 5.2. Material properties gathered from Williams et al. [34] and Michael Stender [32] were assigned to each layer.

Table 5.3. Material properties for Heterogeneous Model Version 2. Values were generated by calculating a 50\% difference between the actual layer and average properties.

Table 7.1. Computational analysis times for the heterogeneous and homogeneous models. Durations for each step are presented to compare (hh:mm:ss).

Table A.1. A 50\% difference from the average value of $\mathrm{E}_{\mathrm{f}}$................................. 74

Table A.2. A 6-layered model displaying variations in $\mathrm{E}_{\mathrm{f}}$.................................... 74

Table A.3. A 10-layered model displaying variations in $\mathrm{E}_{\mathrm{f}}$, ............................... 75 


\section{List of Figures}

Figure 2.1. Each constituent of the SM undergoes an initial deformation to create a stress free SM element. To balance the PG swelling stress, the COL and MAT constituents develop a tensile stress to achieve the stressfree state.

Figure 3.1. A diagram of the motion of a body B. With respect to the origin $\mathbf{O}$, a point $\mathrm{P}$ in the body is tracked with position vectors $\boldsymbol{X}$ and $\boldsymbol{x}$ in the reference and current configurations, respectively.

Figure 4.1. Flow chart of how a UMAT in ABAQUS processes the stress at the next time increment based on the material's constitutive stress equation and forms the stiffness matrix DDSDDE.

Figure 4.2. A representation of the quarter disc model created in ABAQUS with labeled axes, the radius (r), and height (h).

Figure 4.2. The permeability data from Table 4.2 was plotted in MATLAB and curve-fitted to determine $\mathrm{M}$. (A) The S-layer had an M value of 10.6 $\left(R^{2}=0.9991\right)$. (B) The M-layer had an $M$ value of $6.1\left(R^{2}=0.9982\right)$.

Figure 4.3. Maximum principal stress plot of an element centroid located at the top of the model in the S-layer. The data points were gathered from the stress relaxation step of the analysis to determine when the disc reached steady-state conditions

Figure 4.4. Maximum principal stress plot of an element centroid located at the top of the model in the S-layer. The data points were gathered from the peak stress values of each cycle during the dynamic compression step to determine when the disc reached steady-state conditions.

Figure 5.1. Biochemical results from $[34,37]$ for $\mathrm{GAG}$ and $\mathrm{COL}$ as a percent of wet weight.

Figure 6.1. Contour plots of the relative fluid velocity magnitude for the heterogeneous (A) and homogeneous (B) models. Results show that high relative fluid velocities exist at the radial periphery of the disc for both models.

Figure 6.2. Contour plots of the Tresca stress for the heterogeneous (A) and homogeneous (B) models. The Tresca stress exceeded the 0.07 MPa trigger value at a majority of the disc except at the radial periphery.

Figure 6.3. Contour plots of the radial strain for the heterogeneous (A) and homogeneous (B) models. It was suggested that radial strain could stimulate COL synthesis and the results are presented here for discussion.. 51 
Figure 6.4. Contour plots of the minimum principal stress for the heterogeneous (A) and homogeneous (B) models.................................. 53

Figure 6.5. Contour plots of the minimum principal strain for the heterogeneous (A) and homogeneous (B) models.................................. 54

Figure 6.6. Contour plots of the maximum principal stress for the heterogeneous (A) and homogeneous (B) models.................................. 55

Figure 6.7. Contour plots of the maximum principal strain for the heterogeneous (A) and homogeneous (B) models................................... 56

Figure 7.1. Binary contour plots of the Tresca stress for the heterogeneous (A) and homogeneous (B) models. The darker regions show where the Tresca stress exceeded the $0.07 \mathrm{MPa}$ trigger value.

Figure A.1. The relative fluid velocity in the z-direction (A) shows a continuous normal velocity across the discontinuous interface of the two layers. The velocity in the x-direction (B) shows a discontinuous tangential velocity across the interface.

Figure A.2. Reaction fluid volume flux (RVF) due to the pore pressure boundary condition on the radial surface ................................................ 70 


\section{Chapter 1}

\section{Introduction}

Articular cartilage (AC) is an avascular tissue that provides a wear-resistant, lowfriction, load-bearing surface in the articulation of synovial joints $[17,18]$. Soft tissues in the body such as AC consist of two main components: an interstitial fluid mostly consisting of water and dissolved solutes and a solid matrix (SM) comprised mainly of collagen and proteoglycans. Studies have shown that cartilage tissue behaves as a biphasic mixture; deformation applied to the tissue induces fluid flow within the solid matrix [26].

High levels of stresses are transmitted through the tissue during joint movement and body weight support. When AC is damaged due to a disease, traumatic injury or arthritis, the individual often experiences pain and immobility of the joint. Arthritis is a debilitating disease that affects many in the United States. According to a report by the Centers for Disease Control and Prevention, arthritis costs the United States $\$ 128$ billion annually in direct and indirect costs [8]. The report also stated that a study conducted from 2007-2009 found that 49.9 million adults above the age of 18 years had self-reported doctor-diagnosed arthritis [8]. Due to limitations in treatment, damage and arthritis typically progresses to the point 
where total joint replacement is necessary. However, it is an invasive and costly procedure $[15,41]$. Alternatives to this would be to repair or restore the AC surface, but this process has been unsuccessful because the engineered tissue lacks the appropriate mechanical and biological properties of AC [4]. A better understanding of $\mathrm{AC}$ and its properties is needed to develop appropriate remedies for AC surface repair or restoration.

The Cal Poly Cartilage Biomechanics Group has been working toward understanding the biomechanical properties of AC during in vitro growth and remodeling [40]. Explants of AC were subjected to mechanical and biochemical stimulation to understand the tissue's response. Dynamic unconfined compression (UCC) is one type of in vitro mechanical stimulation that is used to understand the response to loading that naturally occurs in vivo [29]. Fluid flow and SM deformation during this loading have biological effects on the health of the tissue $[17,33]$. Building a poroelastic finite element model to mimic the behavior of AC during dynamic UCC provides a framework to design and refine future experimental protocols.

There were three primary aims of this work. The first aim was to develop a finite element analysis (FEA) model of an AC explant that incorporated poroelasticity and a distributed collagen fiber model [32]. The second aim was to analyze physical mechanisms that govern the in vitro growth and remodeling through cellular metabolism and compare them to experimental data ${ }^{1}$ [37]. Third, was to

\footnotetext{
${ }^{1}$ Kevin Yamauchi provided the biochemical data for dynamic UCC.
} 
conduct a feasibility study of modeling a heterogeneous tissue with varying biochemical and mechanical properties [34]. 


\section{Chapter 2}

\section{Background Review}

A biphasic mixture model is adopted to model AC in FEA. Biphasic mixture theory specifies a continuous distribution of solid and fluid phases. The solid phase for $\mathrm{AC}$ is modeled as a solid matrix, comprised of collagen (COL), proteoglycans (PG), and a ground substance matrix (MAT). The fluid phase is comprised of mainly water and dissolved ions [25]. It is assumed that both phases are intrinsically incompressible and the fluid phase is inviscid [14].

The first constituent of the SM is PG, which is a macromolecule consisting of many glycosaminoglycan $(\mathrm{GAG})^{2}$ chains, which are negatively charged [22]. The hydrophilic, highly charged nature of PG within the SM impedes fluid flow and provides resistance to compressive loads that $\mathrm{AC}$ experiences [22]. A swelling pressure is generated as an osmotic pressure that arises due to the fixed negative charge [5].

The second constituent is COL, which is highly organized in the SM and consists mainly of type II collagen. This enables AC to withstand high tensile stresses

\footnotetext{
${ }^{2}$ For the purposes of this analysis, GAG and PG are used interchangeably.
} 
during joint loading [22]. The organization of COL has a depth-dependent nature, where the superficial zone has fibrils aligned tangentially to the surface, the middle zone contains randomly oriented fibrils, and the deep zone contains fibrils oriented perpendicular to the subchondral surface to anchor the tissue to bone [22].

The third constituent is the all-encompassing MAT consisting of chondrocytes and other SM components that have not been accounted for other than PG and COL $[12,21]$. The mechanics of the MAT material is modeled after an isotropic hyperelastic compressible Neo-Hookean material [32].

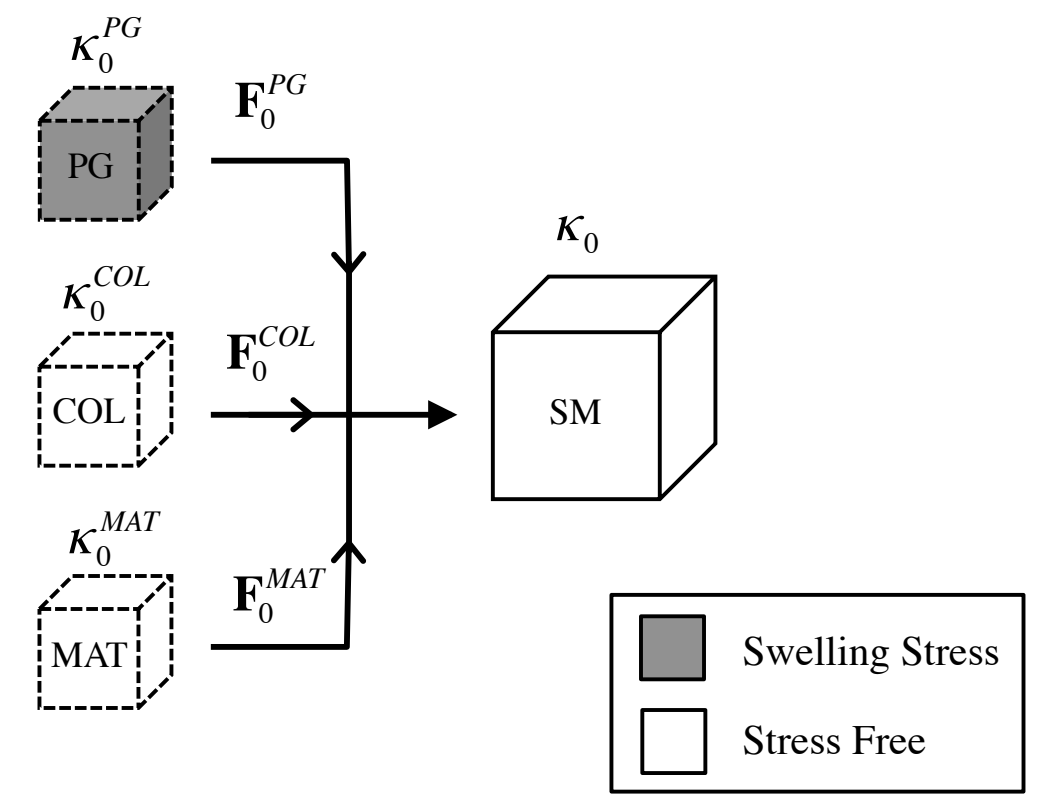

Figure 2.1. Each constituent of the SM undergoes an initial deformation to create a stress free SM element. To balance the PG swelling stress, the COL and MAT constituents develop a tensile stress to achieve the stress-free state.

Each constituent of the SM has an initial reference configuration: $\kappa_{0}^{P G}, \kappa_{0}^{C O L}$, and $\kappa_{0}^{M A T}$. Due to the PG swelling stress in its reference configuration, each 
constituent will undergo initial deformations to develop the stress free SM element (see Figure 2.1). To balance the non-zero GAG stress, the COL and MAT constituents develop a tensile stress to achieve a stress-free SM reference configuration $[19,32,47]$.

Due to the high water content of the material, the interstitial fluid plays a vital role in the tissue response [22]. The fluid provides lubrication of the joints and transports nutrients to the avascular tissue. Compression of the SM induces fluid flow [26]. An increase in compressive strain decreases the permeability, a material's ability to allow fluid to flow, due to the compaction of pores in the material [22].

Studies have shown that material properties of AC are depth dependent $[7,22,34]$. Complex materials such as AC do not exist as a default material in ABAQUS and are introduced through a user-material subroutine (UMAT) that contains a constitutive material model. The UMAT provided the ability to define different COL, GAG, and MAT properties as they varied through the depth of the tissue model. Michael Stender developed the constitutive equations for the UMAT and the corresponding material properties by fitting them to experimental data from [34] and [39]. In these studies, untreated, Day 0 (D0) AC cylindrical explants (discs) were subjected to confined compression, unconfined compression, and tension tests to determine their mechanical properties and then examined for biochemical data. Depth dependent properties for the disc were determined by splitting each disc into two layers: a superficial (S) layer and a middle (M) layer. 
Stender's analysis only studied a single element cube to determine the material constants of the SM.

Studies have subjected AC to many types of mechanical loading protocols to better understand the mechanobiological response such as relations between physical stimuli and cellular metabolism [17]. Under dynamic UCC, gradients in stresses, strains, hydrostatic pressure, and fluid flow are produced in the SM. Low amplitude (1-3\% strains), low frequency $(0.01-0.1 \mathrm{~Hz})$ cyclic loading of AC causes fluid to flow in the tissue and subsequently stimulates PG synthesis [6]. Ficklin [10] developed different models of AC growth based on trigger values such as diffusive fluid velocity and shear stress, but the tissue was only subjected to steady-state fluid permeation and not dynamic UCC loads like those in [6]. Also, the data he modeled came from the middle zone of $\mathrm{AC}$ and in effect he created a homogeneous finite element model. His analysis consisted of a COL bimodular equation, where Stender utilized a new isotropic COL fiber distribution model presented in [30].

Previous studies that developed finite element models (FEM) of AC assumed homogeneous properties. AC response related to joint contact mechanics was analyzed by [36], but constant permeability was assumed during the indentation tests that were modeled. Another joint contact study [48] examined the tissue's stress relaxation and creep response, but only modeled a linear elastic SM with homogeneous strain-dependent permeability. A nonhomogeneous, poroelastic, COL fiber reinforced model was created by [23], but it did not incorporate the PG swelling stress and did not examine the mechanobiological response under 
dynamic UCC. Stender's work is extended to model a disc under dynamic UCC that incorporates fluid flow and heterogeneities to examine the mechanobiological response of AC. 


\section{Chapter 3}

\section{Theory}

\subsection{Kinematics}

Consider a continuous body $B$ that occupies a reference configuration $\kappa_{\mathrm{o}}(\mathrm{B})$ at a time $t=0$ (see Figure 3.1). At a subsequent time t, B occupies a new space called the current configuration $\kappa(B)[31]$.

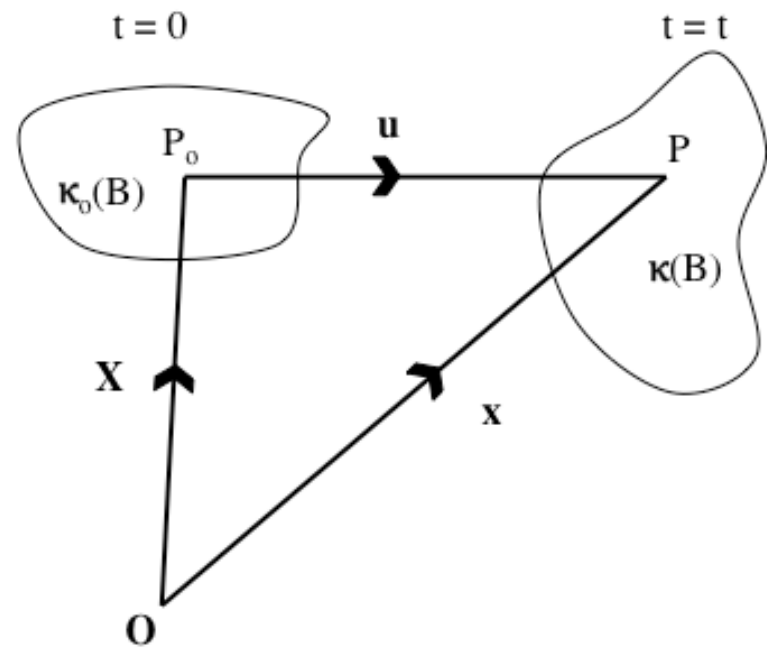

Figure 3.1. A diagram of the motion of a body B. With respect to the origin $\mathbf{O}$, a point $\mathrm{P}$ in the body is tracked with position vectors $\boldsymbol{X}$ and $\boldsymbol{x}$ in the reference and current configurations, respectively. 
A specific point $\mathrm{P}_{\mathrm{o}}$ is located on $\mathrm{B}$ and can be described with position vector $\boldsymbol{X}$ at time $\mathrm{t}=0$. At time $\mathrm{t}$, the same point in the current configuration (denoted by $\mathrm{P}$ ) can be described with position vector $\boldsymbol{x}$. Therefore, the motion of B can be described as

$$
\boldsymbol{x}=\chi(\boldsymbol{X}, t)
$$

and the displacement of this point from its reference to current configuration can be described by the vector

$$
u=x-X .
$$

The deformation gradient tensor is defined as

$$
\boldsymbol{F}=\frac{\partial \chi(\boldsymbol{X}, t)}{\partial \boldsymbol{X}}
$$

and the volumetric changes of B are described by the determinant of $\boldsymbol{F}$ and is given by

$$
J=\operatorname{det} \boldsymbol{F},
$$

where $J$ is referred as the Jacobian. Applying the polar decomposition theorem, $\boldsymbol{F}$ can be described in the form:

$$
\boldsymbol{F}=\boldsymbol{R} \boldsymbol{U}=\boldsymbol{V} \boldsymbol{R},
$$

where $\boldsymbol{R}$ is a proper orthogonal rotation tensor, and $\boldsymbol{U}$ and $\boldsymbol{V}$ are symmetric positive definite right and left-stretch tensors, respectively. The right and left Cauchy-Green deformation tensors ( $\boldsymbol{C}$ and $\boldsymbol{B}$, respectively) are closely related to their respective stretch tensors and the deformation gradient tensor as follows: 


$$
\begin{aligned}
& \boldsymbol{C}=\boldsymbol{F}^{T} \boldsymbol{F}=\boldsymbol{U}^{2}, \\
& \boldsymbol{B}=\boldsymbol{F} \boldsymbol{F}^{T}=\boldsymbol{V}^{2},
\end{aligned}
$$

where $\boldsymbol{C}$ and $\boldsymbol{B}$ are also both symmetric positive definite. The Lagrangian strain tensor is defined as

$$
\boldsymbol{E}=\frac{1}{2}(\boldsymbol{C}-\boldsymbol{I})
$$

\subsection{Biphasic Mixtures}

The theory for biphasic mixtures concerning cartilage is provided in references $[14,20]$ and is summarized below. To symbolize the solid and fluid phases, equations contain superscripts $s$ and $f$, respectively. The intrinsic incompressibility constraint provides the following relationship of the fluid and solid volume fractions:

$$
\phi^{s}+\phi^{f}=1
$$

The constituent volume fractions are defined as $\phi^{\alpha}=\rho^{\alpha} / \rho^{\alpha \mathrm{T}}$, where $\rho^{\alpha}$ is the apparent density and $\rho^{\alpha \mathrm{T}}$ is the true density. The apparent density is the constituent mass divided by the tissue volume and the true density is the constituent mass divided by the constituent volume. Equation (3.9) is used to reduce the continuity equation to: 


$$
\operatorname{div}\left(\phi^{s} \boldsymbol{v}^{s}+\phi^{f} \boldsymbol{v}^{f}\right)=0
$$

where $\boldsymbol{v}$ is the absolute velocity vector of each constituent and $\operatorname{div}()$ is the divergence operator.

The difference between the fluid and solid velocities can be presented as the permeation velocity,

$$
\boldsymbol{v}^{f / s}=\boldsymbol{v}^{f}-\boldsymbol{v}^{s}
$$

The permeation velocity is also known as the relative or effective fluid velocity and reduces to just the fluid phase velocity during steady-state permeation, where the solid phase velocity is zero.

The total Cauchy stress for both phases is

$$
\boldsymbol{T}=\boldsymbol{T}^{s}+\boldsymbol{T}^{f}
$$

where the constituent Cauchy stresses are defined per tissue unit area. The total Cauchy stress $(\boldsymbol{T})$ is also equivalent to the effective stress defined in ABAQUS [9] as

$$
\overline{\boldsymbol{\sigma}}^{*}=\overline{\boldsymbol{\sigma}}+\chi u_{w} \boldsymbol{I}
$$

where $\overline{\boldsymbol{\sigma}}$ is the effective stress of the porous material skeleton $\left(\boldsymbol{T}^{s}\right)$. The second term is equal to $\boldsymbol{T}^{f}$, where $\chi$ is the saturation and varies between 1.0 when the 
medium is fully saturated and 0.0 when the medium is unsaturated, and $u_{w}$ is the wetting liquid (pore) pressure.

The porosity $(n)$ measures the void spaces in a material and is defined by

$$
n=\frac{d V_{v}}{d V}=\frac{e}{1+e}
$$

where $d V_{v}$ is the void volume and $d V$ is the total volume of the material. The void ratio $(e)$ relates the fluid and solid volume fractions and is given by

$$
e=\frac{\phi^{f}}{\phi^{s}}
$$

The material's permeability $(k)$ that $[14]$ derived is presented as

$$
k=k_{o}\left[\frac{\phi_{o}^{S} \phi^{f}}{\left(1-\phi_{o}^{S}\right) \phi^{s}}\right]^{\kappa} \exp \left\{M\left(\lambda^{2}-1\right) / 2\right\}
$$

where $k_{o}$ is the material permeability at zero strain, $\phi_{o}^{s}$ is the initial volume fraction of the solid phase, $\kappa$ is a positive parameter that signifies the rate at which the permeability reaches zero as the solid volume fraction approaches one, $\mathrm{M}$ is a non-dimensional permeability constant, and $\lambda$ is the stretch of the material. Equation (3.16) is for 1-D strains, which exist in confined compression, where $J=\lambda$. The continuity equation for the solid phase is converted into

$$
J \phi^{s}=\phi_{o}^{s}
$$


by dividing both sides of the equation by its true density. Using the relationships given in Equations (3.9) and (3.15), Equation (3.17) can be introduced into Equation (3.16) as

$$
J=\frac{1+e}{1+e_{o}}
$$

where $e_{o}$ is the initial void ratio. Inserting Equation (3.18) into Equation (3.16) and further conversions of the volume fractions into void ratios produces a straindependent permeability function similar to the one presented in [11].

$$
k=k_{o}\left[\frac{e}{e_{o}}\right]^{2} \exp \left[\frac{M}{2}\left(\left\{\frac{1+e}{1+e_{o}}\right\}^{2}-1\right)\right]
$$

\subsection{Constitutive Stress Equations}

The solid matrix (SM) of AC contains three main constituents: PG (GAG), COL, and MAT materials that each has a defined Cauchy stress equation and material Jacobian matrix. The material Jacobian matrix $\left(\widetilde{\mathbb{C}}_{i j k r}^{J a c}\right)$ is required in ABAQUS and is used in the UMAT to guide the computational solver during iterations. All of the constitutive equations in this section were developed in previous studies [30, 32].

Shirazi et al. have developed a model for COL that defines a local unit sphere at each material point, divided into pyramid elements where each contains a volume fraction of COL fibers [30]. A continuous distribution of fibers with an isotropic 
fibril distribution is described as the fibril volume distribution function and is defined as

$$
R(\theta, \Phi)=\frac{\Phi_{n}^{C O L}}{V_{\text {total }}}
$$

where $\Phi_{n}^{C O L}$ is the COL volume fraction of the fibrils in the direction $\boldsymbol{n}$. The total COL volume fraction at a material point is determined by integrating the fibril volume distribution function over the volume of the unit sphere:

$$
\int_{\theta=0}^{\pi} \int_{\varphi=0}^{2 \pi} R(\theta, \Phi) \frac{1}{3} \sin \theta d \theta d \varphi
$$

Due to the tensile load carrying nature of COL the COL fibers do not support compressive stress. The Heaviside step function is defined as

$$
H\left(\boldsymbol{E}_{n}\right)= \begin{cases}0, & \boldsymbol{E}_{\boldsymbol{n}}<0 \\ 1, & \boldsymbol{E}_{\boldsymbol{n}} \geq 0\end{cases}
$$

where $\boldsymbol{E}_{\boldsymbol{n}}$ is the components of Lagrangian stain in the direction of a fiber.

The equation for the Second Piola-Kirchoff COL stress is defined as:

$$
\begin{gathered}
\boldsymbol{S}^{C O L}=\int_{\theta=0}^{\pi} \int_{\varphi=0}^{2 \pi} R(\theta, \Phi)\left(\boldsymbol{E}_{\boldsymbol{n}}\right) H\left(\boldsymbol{E}_{\boldsymbol{n}}\right)\left[\frac{E_{f}}{2}[\boldsymbol{n} \cdot[(\boldsymbol{C}-\boldsymbol{I}) \cdot \boldsymbol{n}]][\boldsymbol{n} \otimes \boldsymbol{n}]\right] \\
\frac{1}{3} \sin \theta d \theta d \varphi,
\end{gathered}
$$

where $E_{f}$ is the true fibril modulus, where the fibril stress-strain relation is linear, $\boldsymbol{n}$ is the unit direction vector, the operator : is the double dot product and $\otimes$ is the 
dyadic product. To transform the second Piola-Kirchoff stress into the Cauchy stress, the following equation is used

$$
J \boldsymbol{T}=\boldsymbol{F} \boldsymbol{S} \boldsymbol{F}^{T} .
$$

The elasticity tensor is related to the Second Piola-Kirchoff stress tensor by:

$$
\mathbb{C}=2 \frac{\partial \boldsymbol{S}}{\partial \boldsymbol{C}}=\frac{\partial \boldsymbol{S}}{\partial \boldsymbol{E}} .
$$

The COL network elasticity tensor is defined as

$$
\mathbb{C}^{C O L}=\int_{\theta=0}^{\pi} \int_{\varphi=0}^{2 \pi} R(\theta, \Phi) H\left(\boldsymbol{E}_{\boldsymbol{n}}\right) E_{f}[[\boldsymbol{n} \otimes \boldsymbol{n}] \otimes[\boldsymbol{n} \otimes \boldsymbol{n}]] \frac{1}{3} \sin \theta d \theta d \varphi
$$

The equations for the MAT material are defined as follows:

$$
\boldsymbol{T}^{M A T}=\frac{1}{J} \boldsymbol{F}\left[\mu\left(\boldsymbol{I}-\boldsymbol{C}^{-1}\right)\right] \boldsymbol{F}^{T},
$$

where $\mu$ is the MAT shear modulus. The MAT material elasticity tensor is

$$
\widetilde{\mathbb{C}}_{A B C D}^{M A T}=\mu\left[C_{A C}^{-1} C_{B D}^{-1}+C_{A D}^{-1} C_{B C}^{-1}\right] .
$$

Michael Stender was able to develop a Cauchy stress function for PG by selecting a suitable PG swelling stress model and fitting it to experimental data. The resulting function is based on the continuum electromechanical PoissonBoltzmann cell model and is presented as

$$
\boldsymbol{T}^{G A G}=-2.87\left(\frac{\rho_{o}^{G A G}}{J}\right)^{2.5} \boldsymbol{I}=-2.87\left(\rho^{G A G}\right)^{2.5} \boldsymbol{I},
$$


where $\rho_{o}^{G A G}$ is the PG apparent density in the reference configuration and $\rho^{G A G}$ is in the current configuration. The PG material elasticity tensor is

$$
\begin{gathered}
\widetilde{\mathbb{C}}_{A B C D}^{G A G}=\left(\frac{\alpha\left(\rho_{o}^{G A G}\right)^{2.5}}{\left(\operatorname{det}\left(\boldsymbol{C}^{G A G}\right)\right)^{1.25}}\right)\left(C_{A C} C_{B D}+C_{A D} C_{B C}\right) \\
+2.5 \frac{\alpha\left(\rho_{o}^{G A G}\right)^{2.5} C_{D C}^{-1} C_{A B}^{-1}}{\left(\operatorname{det}\left(\boldsymbol{C}^{G A G}\right)\right)^{1.25}}
\end{gathered}
$$

For each constituent, the material elasticity tensor and the Cauchy stress tensor are inserted into the following equation:

$$
\begin{aligned}
\tilde{\mathbb{C}}_{i j k r}^{J a c}=\frac{1}{J} \mid\left[\frac { J } { 2 } \left(\delta_{i k} \delta_{p r}\right.\right. & \left.\left.+\delta_{p k} \delta_{i r}\right) T_{p j}+\frac{J}{2}\left(\delta_{j k} \delta_{n r}+\delta_{n k} \delta_{j r}\right) T_{i n}\right] \\
& \left.+\left[\widetilde{\mathbb{C}}_{A B C D} F_{i A} F_{j B} F_{k C} F_{r D}\right]\right]
\end{aligned}
$$

and then they are summed together to form the total material Jacobian matrix. 


\section{Chapter 4}

\section{Implementation}

\subsection{UMAT}

A user-material subroutine (UMAT) is used in ABAQUS when a specific material and its mechanical constitutive behavior are undefined in the program. Complex materials such as articular cartilage tissue are non-linear and contain multiple components such as PG, COL, and MAT. A UMAT allows the user to define these complex materials through mechanical constitutive equations. The subroutine is written in Fortran, which is a numeric computation programming language.

\subsubsection{ABAQUS Implementation}

ABAQUS (v6.9-2) uses the UMAT to update stresses and solution-dependent state variables at every increment using the Jacobian stiffness matrix (DDSDDE), 
which assists with convergence [9]. The material properties used to describe the constitutive behavior of the cartilage are passed to the UMAT depending on the material point that ABAQUS is analyzing. At a previous time $t_{n}$, ABAQUS passes to the UMAT the values of the stress at that time $\sigma\left(t_{n}\right)$, the time increment $\Delta \mathrm{t}$, the deformation gradient tensor $\mathbf{F}\left(\mathrm{t}_{\mathrm{n}}\right)$, a guess for the deformation gradient tensor for the current time $\mathbf{F}\left(\mathrm{t}_{\mathrm{n}+1}\right)$, and any defined material variables. The UMAT calculates the new Cauchy stress and provides the stiffness matrix DDSDDE from the given information. ABAQUS then makes a new guess for $\mathbf{F}$. This process is iterated until convergence (see Figure 4.1) [38].

ABAQUS

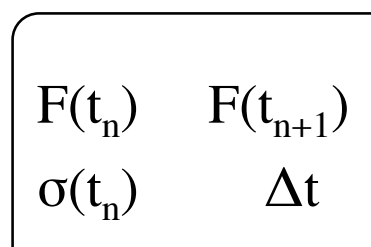

UMAT

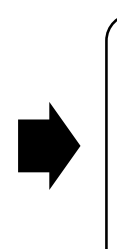

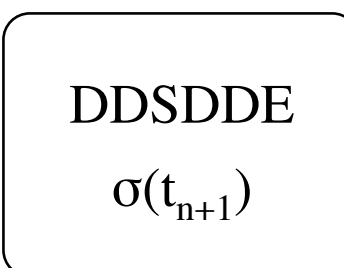

ABAQUS

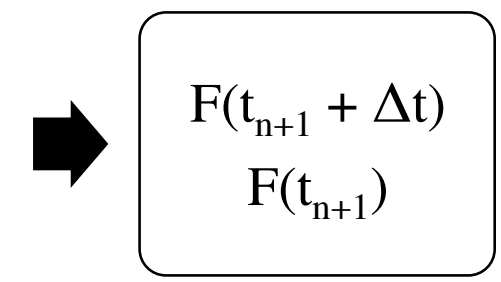

Figure 4.1. Flow chart of how a UMAT in ABAQUS processes the stress at the next time increment based on the material's constitutive stress equation and forms the stiffness matrix DDSDDE.

For biphasic theory, the UMAT is only concerned with the definition of the solid matrix properties and its Cauchy stress $\left(\mathbf{T}^{\mathrm{SM}}\right)$. The fluid stress and pore pressure are not defined in the UMAT and are handled in ABAQUS. 


\subsubsection{Michael Stender's UMAT}

The UMAT that Michael Stender created was used in its entirety for these analyses. The Cauchy stress tensor needed for the solid matrix was defined from the stress balance hypothesis [3],

$$
\boldsymbol{T}^{S M}=\boldsymbol{T}^{P G}+\boldsymbol{T}^{C O L}+\boldsymbol{T}^{M A T},
$$

where it was equal to the sum of all the constituents. Each of the constituent Cauchy stress tensors was previously defined in the Theory section above. The UMAT that Stender created allowed the user to define material values for different layers of the articular cartilage tissue (superficial or middle layer). Stender gathered these properties from experimental data, which was also used to fit parameters in defining the correct Cauchy stress functions for each of the constituents [32].

\subsection{ABAQUS Modeling}

In $\mathrm{ABAQUS} / \mathrm{CAE}^{3}$ (v6.9-2), unconfined compression of a quarter disc was modeled with appropriate boundary conditions to reduce the computation time. The dimensions of the disc mimicked the experiments that Kevin Yamauchi [37] conducted and consisted of a $1.25 \mathrm{~mm}$ radius and a $1.15 \mathrm{~mm}$ initial height $\left(\mathrm{h}_{\mathrm{o}}\right)$.

\footnotetext{
${ }^{3}$ The visualization program of ABAQUS is called ABAQUS/CAE (computer aided engineering), which used to develop the analysis models.
} 
The AC explants were subjected to an initial $10 \%$ compressive strain followed by a stress relaxation stage and then by a $0.1 \mathrm{~Hz}$ periodic displacement of $\pm 2 \%$ strain.

\subsubsection{Meshing}

To create a mesh of 960 elements, a seed number of 10 elements was specified along the radius and height of the quarter disc (See Figure 4.2). Mesh controls were set to a hexahedral shape using the structured technique. This analysis used a C3D8P element, which is an 8-node brick with trilinear displacement and trilinear pore pressure.

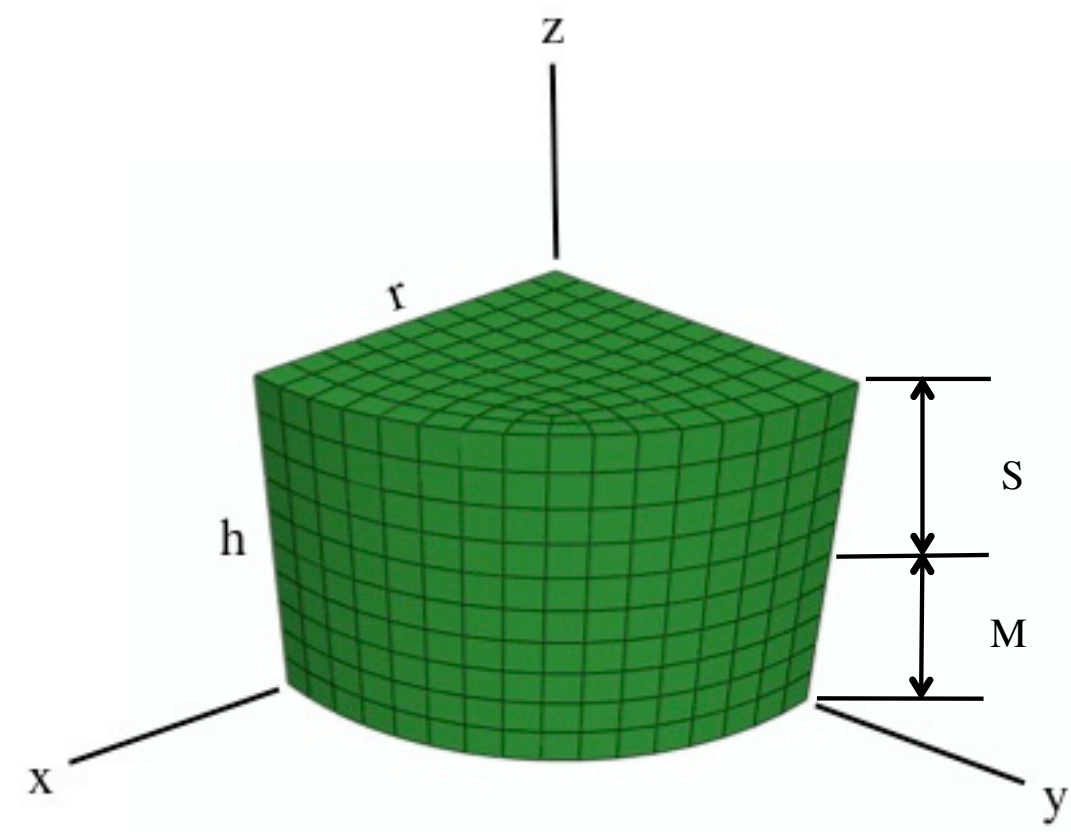

Figure 4.2. A representation of the quarter disc model created in ABAQUS with labeled axes, the radius ( $\mathrm{r}$ ), and height (h). 


\subsubsection{Material Properties}

Material properties were set for each of the superficial (S) and middle (M) layers with poroelastic and UMAT definitions. The layers had equivalent initial heights of $0.575 \mathrm{~mm}$ (See Figure 4.2).

\subsubsection{User Material Properties}

Material constants for each layer that were used in the UMAT are set under the User Material section of the General tab in ABAQUS/CAE. ABAQUS passes the UMAT the PROPS(x) vector depending on what material point is being analyzed. Minor adjustments to Stender's UMAT [32] were made to allow ABAQUS to pass these values per layer. Table 4.1 displays the material constants, where PROPS(1) corresponds to the first listed value that is entered in ABAQUS.

Table 4.1. Material property variables set in the UMAT for the S and M layers.

\begin{tabular}{|c|c|l|}
\hline PROPS(x) & Variable & \multicolumn{1}{|c|}{ Description } \\
\hline 1 & $\mathrm{E}_{f}$ & COL fiber modulus \\
\hline 2 & $\Phi_{f}$ & COL volume fraction \\
\hline 3 & $\rho_{o}^{P G}$ & $\begin{array}{l}\text { Reference configuration } \\
\text { GAG density per wet weight }\end{array}$ \\
\hline 4 & $\mu$ & MAT shear modulus \\
\hline
\end{tabular}

\subsubsection{Permeability}

ABAQUS utilizes the permeability by selecting it from a user-defined look-up table that lists the permeability as a function of void ratio. To develop this function, Equation (3.19) is manipulated to be a function of strain $(\varepsilon)$ and initial 
void ratio $\left(e_{o}\right)$. By letting $\lambda=1+\varepsilon$ and recalling Equation (3.18), a relationship between void ratio and strain is

$$
e=(\varepsilon+1)\left(1+e_{o}\right)-1
$$

Using Equation (4.2) in Equation (3.19), defines the permeability as

$$
k=k_{o}\left[\frac{(\varepsilon+1)\left(1+e_{o}\right)-1}{e_{o}}\right]^{2} \exp \left[\frac{M}{2}\left(\varepsilon^{2}+2 \varepsilon\right)\right]
$$

To determine the strain-dependent permeability function for each layer of the cartilage, the experimental data from [34] was fitted to Equation (4.3). The data in Table 4.2 provided permeability values that were measured for each layer while the tissue was subjected to different $\mathrm{CC}$ strain ramps. The data was plotted in MATLAB and the curve-fitting tool was used to determine the non-dimensional permeability constant, $M$ (See Figure 4.3). The initial permeability $k_{o}$ is the permeability value at $0 \%$ strain. The initial void ratios $\left(e_{o}\right)$ from the experimental data for the superficial (S) and middle (M) layers were 8.381 and 6.479, respectively. The $\mathrm{M}$ values for the superficial and middle layers were 10.6 and 6.1 , respectively.

Table 4.2. Experimental permeability values $\left[\mathrm{m}^{2} / \mathrm{Pa}-\mathrm{s}\right]$ for the $\mathrm{S}$ and $\mathrm{M}$ layers at different CC strains [34].

\begin{tabular}{|c|c|c|c|c|}
\hline Layer & $\mathbf{0 \%}$ & $\mathbf{- 1 5 \%}$ & $\mathbf{- 3 0 \%}$ & $\mathbf{- 4 5 \%}$ \\
\hline $\mathbf{S}$ & $9.83 \mathrm{E}-15$ & $1.33 \mathrm{E}-15$ & $3.65 \mathrm{E}-16$ & $9.16 \mathrm{E}-17$ \\
\hline $\mathbf{M}$ & $2.02 \mathrm{E}-15$ & $6.27 \mathrm{E}-16$ & $1.24 \mathrm{E}-16$ & $5.87 \mathrm{E}-17$ \\
\hline
\end{tabular}


(A)

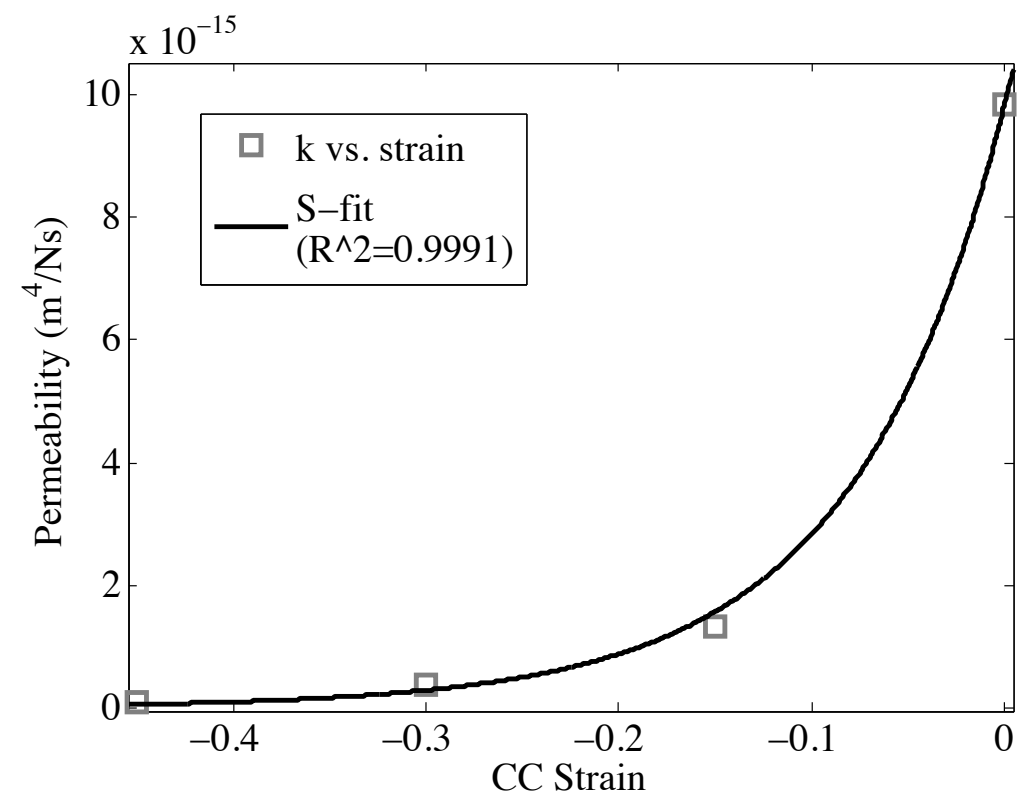

(B)

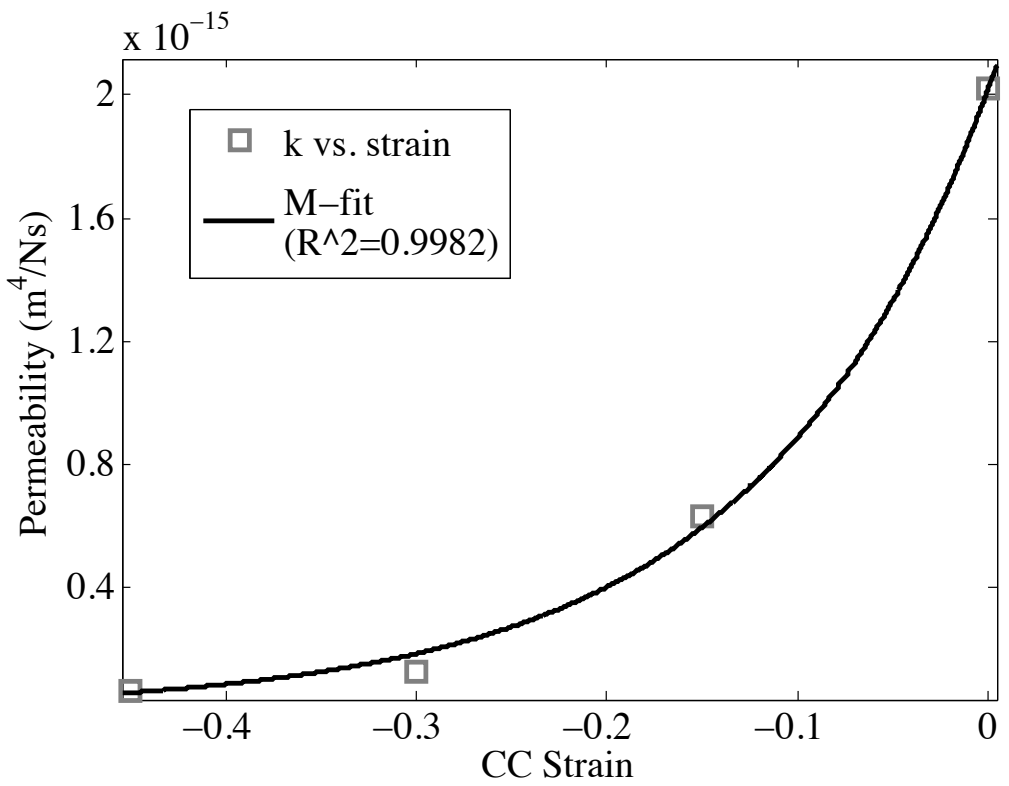

Figure 4.3. The permeability data from Table 4.2 was plotted in MATLAB and curve-fitted to determine M. (A) The S-layer had an $M$ value of $10.6\left(R^{2}=0.9991\right)$. (B) The M-layer had an $\mathrm{M}$ value of $6.1\left(\mathrm{R}^{2}=0.9982\right)$. 
The permeability look-up table was created in Excel using Equation (3.19) with the corresponding $\mathrm{M}$ values. The resolution of the void ratio in the lookup table was 0.001 to account for small strain increments used in the analysis.

Permeability is measured in units of $\frac{\mathrm{m}^{2}}{\mathrm{~Pa} \cdot \mathrm{s}}$ which relates the fluid velocity to a pressure gradient that is applied to the SM. Darcy's Law is given as

$$
Q=\frac{k}{\mu} \frac{A \Delta P}{L}
$$

where $Q$ is the volumetric flow rate, $A$ is the cross-sectional area of the flow, $\Delta P$ is the pressure drop, and $L$ is the length of the pressure drop. Due to the assumption that the fluid phase is inviscid, $\mu$ is neglected and solving for $k$ gives

$$
k=\frac{Q L}{A \Delta P}\left[\frac{m^{2}}{P a \cdot s}\right]
$$

ABAQUS uses the hydraulic conductivity $\left(k_{A}\right)$, which is related to the permeability by

$$
k_{A}=k \gamma_{w}\left[\frac{m m}{s}\right]
$$

where the permeability $(\mathrm{k})$ is multiplied by the specific weight of the wetting liquid $\left(\gamma_{w}\right)$. The specific weight of the wetting liquid is $9.741 \times 10^{-6} \mathrm{~N} / \mathrm{mm}^{3}$ and is assumed to be water at body temperature. 


\subsubsection{Node and Element Sets}

Node and element sets of the quarter disc model were created to assign properties, initial conditions and boundary conditions. Although the user can assign these definitions directly in the viewport, creating sets allows jobs to run on remote computers using an input file that ABAQUS/CAE generates. By assigning sets, the input file can easily determine what elements or nodes to select. If they were simply selected through the viewport in ABAQUS/CAE (picked set), other computers would not recognize the nodes or elements selected in the picked set.

Element sets were created for: all elements of the quarter disc, the S-Layer (top half), and the M-Layer (bottom half). Node sets were created for: all nodes of the quarter disc, the bottom surface, the top surface, the $\mathrm{Y}-\mathrm{Z}$ plane, the $\mathrm{X}-\mathrm{Z}$ plane, the outer radial surface, all nodes contained in the S-Layer, all nodes contained in the M-Layer, and the nodes at the interface of the two layers.

Sections were created with each of the layer material properties and were assigned to the corresponding element sets (S or M layer).

\subsubsection{Analysis Steps}

Steps were generated in ABAQUS/CAE that simulated the dynamic loading experimental conditions. Non-linear geometry was used for all steps because large deformations were applied to the disc. Incrementation parameters are assigned in 
this section of ABAQUS/CAE (See Table 4.3). The first step was a static equilibrium step that allows the disc to swell and the subsequent steps are SOILS analysis steps that are used for biphasic materials and account for the pore fluid response and permeability.

Table 4.3. The time periods and incrementation sizes chosen for the analysis steps.

\begin{tabular}{|c|c|c|c|c|c|}
\hline & \multicolumn{4}{|c|}{ Analysis Steps } \\
\hline & & $\begin{array}{c}\text { Static } \\
\text { Equilibrium }\end{array}$ & $\begin{array}{c}\text { Initial } \\
\text { Compression }\end{array}$ & $\begin{array}{c}\text { Stress } \\
\text { Relaxation }\end{array}$ & $\begin{array}{c}\text { Dynamic } \\
\text { Displacement }\end{array}$ \\
\hline \multicolumn{2}{|c|}{ Time period (s) } & 1 & 115.5 & 1500 & 50 \\
\hline \multirow{3}{*}{ 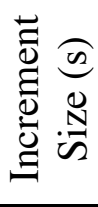 } & Initial & - & 1 & 100 & 0.5 \\
\hline & Minimum & - & $1.00 \mathrm{E}-05$ & 0.01 & 0.05 \\
\hline & Maximum & - & 25 & 500 & 0.5 \\
\hline
\end{tabular}

\subsubsection{Static Equilibrium}

PG contains a swelling stress that causes the cartilage material to be in a state of nonzero stress in the reference configuration. To account for this, the quarter disc was first run through a static equilibrium step that allows the disc to swell. ABAQUS deforms the SM until it reaches a stress free configuration. Because this was a static equilibrium step, the pore fluid response was not incorporated into this portion of the analysis. Static equilibrium is reached at every increment, and therefore, this step was fixed to a single increment to find the equilibrium height, $h_{e}$. This step was run alone to determine $h_{e}$ and then applied to the subsequent steps of compressive strain, which was based on this height. 


\subsubsection{Initial Compression}

The Initial Compression step, along with the subsequent steps, was run using the SOILS analysis in ABAQUS because it accounts for the pore pressure response and permeability. The unconfined compressive displacement during this step was a $10 \%$ strain calculated from the equilibrium height $h_{e}$, which was determined in the previous step. Experimentally, the disc was loaded at a rate $\mathrm{v}_{\mathrm{o}}=0.001 \mathrm{~mm} / \mathrm{s}$. The time period $(\mathrm{t})$ for this simulation step was calculated by dividing the displacement by the loading rate, $\mathrm{t}=0.1 \mathrm{~h}_{\mathrm{e}} / \mathrm{v}_{\mathrm{o}}$. Table 4.3 contains the time period and incrementation size for this step. Because the information during this step was not of interest, the increment size was chosen to move through the entire time period quickly. For SOILS analyses, a tolerance for maximum pore pressure change per increment was required. This was set to 0.1 , an arbitrary non-zero value that allows for automatic incrementation.

\subsubsection{Stress Relaxation}

Experimentally, the cartilage disc was allowed to relax for 1200 s, which enabled it to return to steady-state conditions after the initial compression. The time period for this step was set to 1500 s, which has an added excess of time for the complexity of the material. Analyses were run to determine that steady-state was reached before the 1500s (Figure 4.4). The incrementation size to determine the time period was fixed to 100 s, so that information could be gathered to show that the disc had equilibrated. 


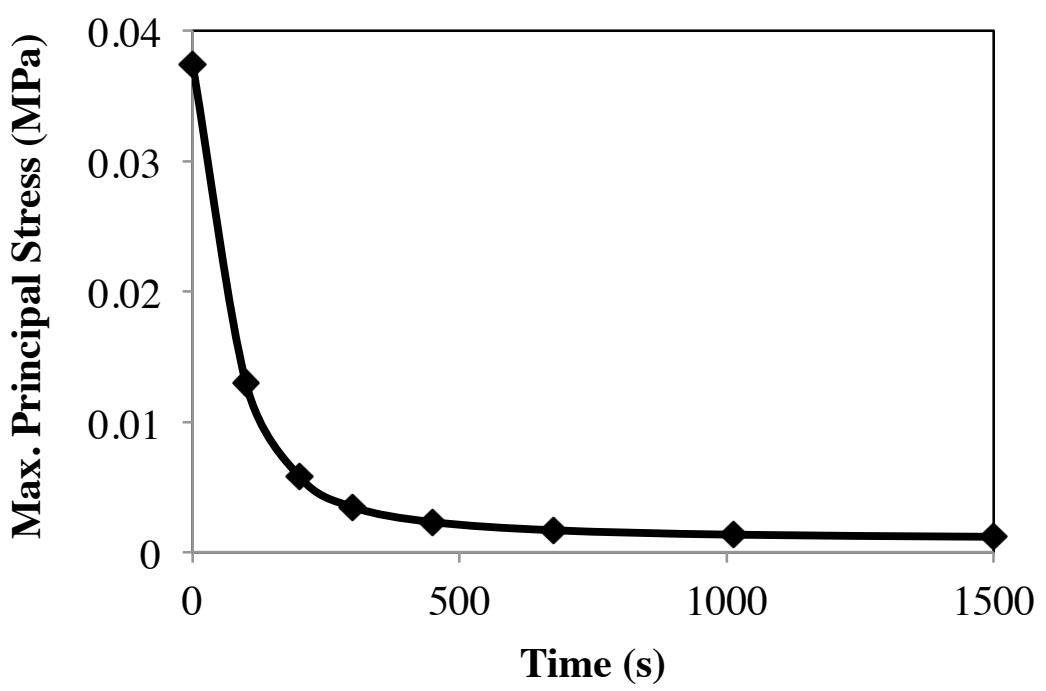

Figure 4.4. Maximum principal stress plot of an element centroid located at the top of the model in the S-layer. The data points were gathered from the stress relaxation step of the analysis to determine when the disc reached steady-state conditions.

\subsubsection{Dynamic Displacement}

The disc was loaded with a sinusoidal displacement of $\pm 2 \%$ strain at a frequency of $0.1 \mathrm{~Hz}$. This dynamic displacement was applied from the $10 \% \mathrm{~h}_{\mathrm{e}}$ initial compression (the resulting amplitude of the periodic displacement was between $8 \% \mathrm{~h}_{\mathrm{e}}$ to $12 \% \mathrm{~h}_{\mathrm{e}}$ ). The time period of this step was set to $50 \mathrm{~s}$, which would allow five full cycles of the displacement to elapse. Previous analyses were run to determine that steady-state conditions were met before results were obtained from the fifth cycle. Steady state during dynamic compression was determined by comparing the peak stresses during each cycle. Figure 4.5 shows that the fifth cycle reached steady state due to the decrease in the change of the peak stresses. The incrementation size for this step is presented in Table $\mathbf{4 . 3}$ and a maximum increment of $0.5 \mathrm{~s}$ was chosen so that ample amounts of data during a cycle could 
be collected for post processing. It was determined that if the incrementation size fell below $0.05 \mathrm{~s}$ at $0.1 \mathrm{~Hz}$, the disc would not experience enough of a displacement. In this case, ABAQUS would diverge because it assumes that there was negligible change to the disc.

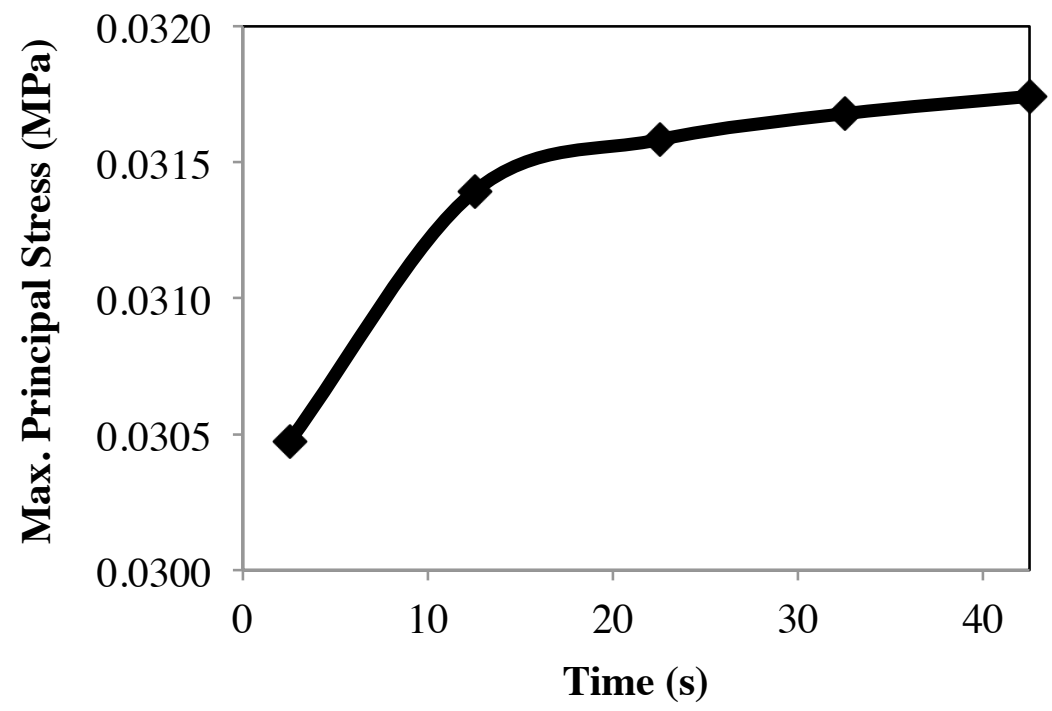

Figure 4.5. Maximum principal stress plot of an element centroid located at the top of the model in the S-layer. The data points were gathered from the peak stress values of each cycle during the dynamic compression step to determine when the disc reached steady-state conditions.

The data that was collected from this step for post processing was selected from the Field Output Requests section in ABAQUS/CAE. The field outputs of interest were:

1. S, stress components and invariants

2. EE, elastic strain components (solid matrix strains)

3. U, translations

4. Porous media/fluids variables: 

a. VOIDR, void ratio
b. FLVEL, pore fluid effective velocity
c. POR, pore pressure
d. RVF, reaction volume flux
e. RVT, reaction total volume

\subsubsection{Boundary Conditions}

In ABAQUS/CAE, the boundary conditions (BCs) are assigned in the Load module. It is important to note that the BCs were assigned to the node sets created previously and not to picked sets created by selecting surfaces through the viewport.

\subsubsection{General Boundary Conditions}

In Figure 4.2, the $\mathrm{X}-\mathrm{Z}$ plane set of nodes was constrained in the $\mathrm{y}$-direction $(\mathrm{U} 2=0)$, the $\mathrm{Y}-\mathrm{Z}$ plane set of nodes was constrained in the $\mathrm{X}$-direction $(\mathrm{U} 1=0)$, and the bottom surface set of nodes was constrained in the $\mathrm{z}$-direction (U3=0).

An additional $\mathrm{BC}$ for pore pressure is assigned to the outer radial surface. Experimentally, the disc is loaded between two impermeable platens and therefore, fluid flow was only allowed through the outer radial surface. To simulate this in ABAQUS/CAE, the pore pressure on this surface is set to zero, which is equivalent to atmospheric pressure. This BC was initialized in the first 
SOILS step (Initial Compression), as the Static Equilibrium step does not include poroelastic effects.

\subsubsection{Initial Compression Boundary Condition}

The BC for the Initial Compression analysis step was applied to the set of nodes on the top surface of the quarter disc. It was initialized in this step and was maintained through the Stress Relaxation step. The $10 \%$ compressive strain $\left(\delta_{10 \%}\right.$ $\left.=0.10 \mathrm{~h}_{\mathrm{e}}\right)$ was based off the equilibrium height $\left(\mathrm{h}_{\mathrm{e}}\right)$, but ABAQUS applies the displacement BCs to the original height $\left(h_{\mathrm{o}}\right)$. The ramp displacement $\mathrm{BC}$ for this step in the axial direction was

$$
U 3=-\left[h_{o}-\left(h_{e}-\delta_{10 \%}\right)\right]
$$

\subsubsection{Dynamic Displacement Boundary Condition}

The dynamic displacement $\mathrm{BC}$ of $\pm 2 \% \mathrm{~h}_{\mathrm{e}}$ was applied to the set of nodes on the top surface during the Dynamic Displacement step. The initial compression BC, defined in the previous section, was removed during this step so it wouldn't conflict with the new BC. The displacement in the z-direction (U3) was defined as

$$
U 3=\delta_{2 \%}=-0.02 h_{e}
$$

which defines the initial amplitude. The amplitude displacement is set to a periodic function and is defined in ABAQUS [9] as 


$$
a=U 3\left\{A_{o}+\sum_{n}^{N}\left[A_{n} \cos n \omega\left(t-t_{o}\right)+B_{n} \sin n \omega\left(t-t_{o}\right)\right]\right\}
$$

where $\mathrm{A}_{\mathrm{o}}$ starts the periodic loading at the initial $10 \%$ compressive strain:

$$
A_{o}=\frac{U 3_{10 \%}}{U 3_{2 \%}} .
$$

Only one iteration of the sine function is used for this periodic loading condition, and therefore, $\mathrm{n}=1, \mathrm{~A}_{\mathrm{n}}=0, \mathrm{~B}_{\mathrm{n}}=1$, and it begins at the start of the analysis step $\mathrm{t}_{\mathrm{o}}$ $=0$. The frequency $(\omega)$ is expressed in radians $/ \mathrm{s}$ and is given as $\omega=0.6283 \mathrm{rad} / \mathrm{s}$ $(0.1 \mathrm{~Hz})$. Equation $(4.10)$ reduces to

$$
a=U 3\left\{A_{o}+\sin \omega t\right\} .
$$

\subsection{Keywords Adjustments}

One of the limitations in ABAQUS/CAE was its ability to define initial conditions (IC) when doing complex analyses. For a poroelastic analysis, the initial void ratio and saturation needed to be defined, but were not supported by ABAQUS/CAE. To work around this, ABAQUS allows the user to make adjustments to the input file under Keyword Adjustments. This displays a truncated input file where the user can add lines of code. A line for each IC was added with the type of IC, the node set it was being assigned, and the value. An initial void ratio (defined previously in Equation (3.15)) was assigned to each of the S-Layer node set, M-Layer node set, and the interface node set (the average of the two layers). The IC for the saturation was assigned to all nodes and had a 
value of 1.0, which indicates that the quarter disc was fully saturated. In addition to the poroelastic ICs, the initial stress was set to zero for all elements. 


\section{Chapter 5}

\section{Experimental Results and FEA Parameters for Dynamic UCC}

The primary aim of this study was to develop a heterogeneous finite element model of articular cartilage and study the tissue response when subjected to a dynamic compression. This chapter will examine the biochemical results from dynamic loading experiments, present the material properties from experimental data used in this analysis, and review the physical mechanisms responsible for biosynthesis in cartilage.

\subsection{Dynamic UCC Experimental Data}

The finite element analysis of the heterogeneous cartilage disc was modeled after dynamic loading experiments that Kevin Yamauchi [37] conducted. The AC discs were extracted from the patellofemoral ridge of an immature bovine stifle joint. They were approximately $2.5 \mathrm{~mm}$ in diameter and $1.15 \mathrm{~mm}$ in height. The explants were loaded between two impermeable platens and were subjected to 
unconfined compression. The loading procedure of this experiment consisted of a ramp to $10 \%$ compressive strain at a rate of $0.001 \mathrm{~mm} / \mathrm{s}$ followed by a stress relaxation stage for $1200 \mathrm{~s}$. A sinusoidal displacement of $\pm 2 \%$ strain was then applied for 8 hours followed by 16 hours of free swelling for six days. The tissue was subjected to dynamic loading for a total of 24 hours. To detect radial differences in biochemical data, each disc was radially punched. The inner core consisted of the radius from $0.00 \mathrm{~mm}$ to $0.75 \mathrm{~mm}$ and the outer ring was from $0.75 \mathrm{~mm}$ out to $1.25 \mathrm{~mm}$.

\subsubsection{Dynamic UCC Experimental Results}

Biochemical data was collected on the inner core and outer ring of five dynamically loaded AC explants [37]. Table 5.1 and Figure 5.1 present data from $\mathrm{S} 10+\mathrm{D}$, which is the $10 \%$ static compression included with the dynamic compression. The D0 data is provided to determine if GAG and COL synthesis occurred by comparing the contents to the $\mathrm{S} 10+\mathrm{D}$ data. The experimental results for D0 specimens came from [34], which is the same source for the material properties used in the FEA. The DO S and M layer GAG and COL contents were averaged and assumed to be the same in the inner and outer regions of the disc because they have not undergone mechanical stimulation. D0 specimens from [37] exist, but data have yet to be collected. The free swelling bioreactor (FSB) results from [37] are also provided for reference in Table 5.1 and Figure 5.1, but 
cell metabolism in the inner core may have been compromised due to the lack of mechanical loading and distance from the surrounding medium to get nutrients to the inner core. Comparison with the D0 data provides much stronger evidence for the occurrence of biosynthesis.

Table 5.1. Experimental results for D0 specimens from [34] and FSB and S10+D data from [37]. Biochemical data is presented for GAG and COL content as a percentage of wet weight.

\begin{tabular}{|c|c|c|c|c|c|}
\hline & \multicolumn{2}{|c|}{ GAG/WW [\%] } & \multicolumn{2}{|c|}{ COL/WW [\%] } \\
\hline & & Mean & Stdev & Mean & Stdev \\
\hline \multirow{3}{*}{$\bar{\theta}$} & $S$ & 3.279 & 0.748 & 5.531 & 1.391 \\
\hline & M & 4.760 & 1.213 & 7.153 & 2.068 \\
\hline & Average & 4.019 & 0.981 & 6.342 & 1.729 \\
\hline \multirow{3}{*}{ 卷 } & Inner & 4.601 & 1.876 & 4.179 & 1.378 \\
\hline & Outer & 6.406 & 3.159 & 5.203 & 4.003 \\
\hline & Whole & 6.077 & 1.822 & 4.781 & 3.364 \\
\hline \multirow{3}{*}{$\stackrel{\oplus}{\stackrel{t}{\theta}}$} & Inner & 5.493 & 0.307 & 5.339 & 1.133 \\
\hline & Outer & 12.987 & 1.739 & 5.152 & 0.693 \\
\hline & Whole & 10.037 & 0.645 & 5.196 & 0.797 \\
\hline
\end{tabular}

Statistical analyses were conducted on the data in Table 5.1 to compare the GAG and COL contents from D0 and S10+D specimens. A two-sample T-test was performed in Minitab with a significance level of 0.05 . The full statistical results are presented in the Appendix. 

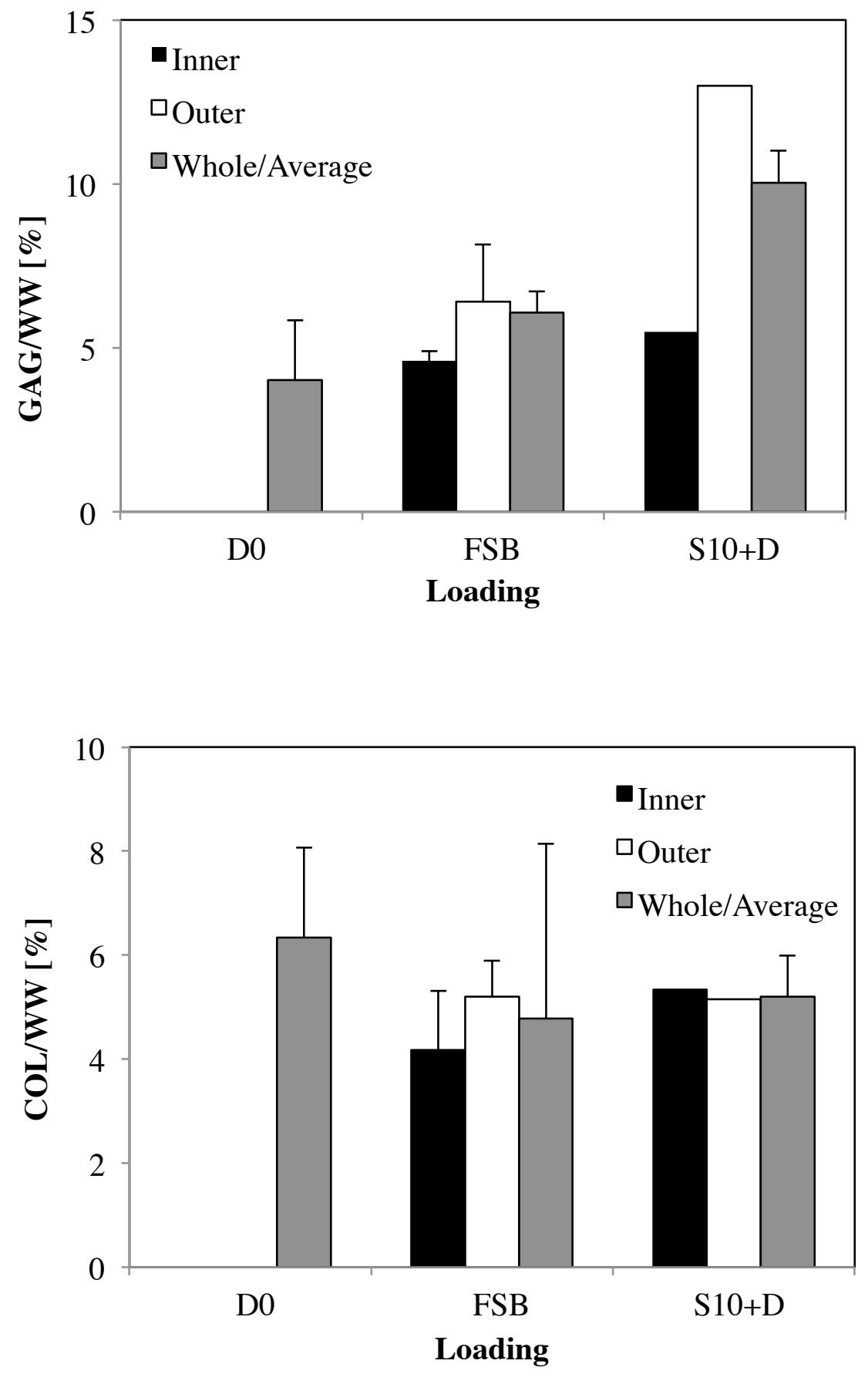

Figure 5.1. Biochemical results from $[34,37]$ for GAG and COL as a percent of wet weight.

The biochemical results in Figure 5.1 and Table 5.1 show that after the dynamic loading process $(\mathrm{S} 10+\mathrm{D})$, concentration of $\mathrm{COL}$ in the inner core was not 
statistically different than that of the outer ring $(\mathrm{P}=0.764)$. Mean COL concentrations for the inner core and outer ring were 5.339 and $5.152 \% \mathrm{WW}$, respectively. Comparing with D0 samples, the S10+D inner core COL contents were also not statistically different $(\mathrm{P}=0.163)$. For the outer ring, $\mathrm{D} 0 \mathrm{COL}$ content was statistically greater than that of the $\mathrm{S} 10+\mathrm{D}$ specimens $(\mathrm{P}=0.019)$ and therefore, COL content may have decreased in the outer regions of the disc. This provides evidence that COL synthesis does not occur during dynamic UCC. The results for the whole discs of S10+D and FSB show that the COL concentrations were not statistically different $(\mathrm{P}=0.391)$.

For S10+D samples, GAG concentration in the inner core was statistically different $(\mathrm{P}<0.001)$ than that of the outer ring, with the outer ring having a higher GAG content. Mean GAG concentrations for the inner core and outer ring were 5.493 and $12.987 \% \mathrm{WW}$, respectively. The S10+D inner core and outer ring GAG concentrations were statistically greater than that of the D0 samples $(\mathrm{P}<0.001)$. This provides evidence that GAG synthesis occurred throughout the disc with statistically greater levels in the outer region (periphery) during dynamic UCC. Comparing with the FSB results, the inner core GAG concentrations were not statistically different, but the S10+D outer ring concentration was statistically greater $(\mathrm{P}<0.002)$. 


\subsection{FEA Material Properties}

\subsubsection{Heterogeneous Model}

A finite element model of a heterogeneous AC disc was created based off of experimental data from Williams et al. [34] as well as constitutive properties and a UMAT that Michael Stender [32] developed. The material properties were collected from two regions of disc explants, labeled the superficial and middle layers. The FEA model was developed such that each of the layers had homogeneous properties, but combined would develop a heterogeneous quarter disc model. The properties for the heterogeneous model are presented in Table 5.2 . 
Table 5.2. Material properties gathered from Williams et al. [34] and Michael Stender [32] were assigned to each layer.

\begin{tabular}{|c|c|c|c|c|}
\hline Material Constant & Units & S-Layer & M-Layer & Average \\
\hline $\mathbf{E}_{\boldsymbol{f}}$ & $\mathrm{MPa}$ & 320 & 550 & 435 \\
\hline $\boldsymbol{\Phi}_{\boldsymbol{f}}$ & - & 0.03787 & 0.04979 & 0.04383 \\
\hline $\boldsymbol{\rho}_{\boldsymbol{o}}^{\boldsymbol{P G}}$ & $\frac{m g}{m l}$ & 3.279 & 4.76 & 4.0195 \\
\hline $\boldsymbol{\mu}$ & $\mathrm{MPa}$ & 0.001 & 0.001 & 0.001 \\
\hline $\mathbf{e}_{\mathbf{o}}$ & - & 8.381 & 6.479 & 7.43 \\
\hline $\mathbf{k}_{\mathbf{o}}$ & $\frac{m^{4}}{N s}$ & $9.83 \mathrm{E}-15$ & $2.02 \mathrm{E}-15$ & $5.925 \mathrm{E}-15$ \\
\hline $\mathbf{M}$ & - & 10.6 & 6.1 & 9.5 \\
\hline $\mathbf{h}_{\mathbf{e}}$ & $\mathrm{mm}$ & \multicolumn{1}{|c|}{1.155} & 1.155 \\
\hline
\end{tabular}

\subsubsection{Heterogeneous Model Version 2}

The material properties assigned in Table $\mathbf{5 . 2}$ caused convergence issues in ABAQUS during the finite element analysis. The change in properties from the $S$ to the M layer was too large for convergence to occur (other possible reasons for convergence issues are mentioned in the Discussion: Feasibility section). A 50\% difference between the actual layer properties and the average properties was the greatest separation in property values that allowed convergence. All material properties were subjected to this $50 \%$ difference except for $\mathrm{M}$, the permeability constant, which was recalculated using the method in Section 4.2.2.2. The new set 
of material properties is listed in Table 5.3. Other attempted methods used to maintain convergence are listed in the Appendix.

Table 5.3. Material properties for Heterogeneous Model Version 2. Values were generated by calculating a $50 \%$ difference between the actual layer and average properties.

\begin{tabular}{|c|c|c|c|}
\hline Material Constant & Units & S-Layer & M-Layer \\
\hline $\mathbf{E}_{\boldsymbol{f}}$ & $\mathrm{MPa}$ & 377.5 & 492.5 \\
\hline $\boldsymbol{\Phi}_{\boldsymbol{f}}$ & - & 0.04085 & 0.04681 \\
\hline $\boldsymbol{\rho}_{\boldsymbol{o}}^{\boldsymbol{P G}}$ & $\frac{m g}{m l}$ & 3.64925 & 4.38975 \\
\hline $\boldsymbol{\mu}$ & $\mathrm{MPa}$ & 0.001 & 0.001 \\
\hline $\mathbf{e}_{\mathbf{o}}$ & - & 7.9055 & 6.9545 \\
\hline $\mathbf{k}_{\mathbf{o}}$ & $\frac{m^{4}}{N s}$ & $7.88 \mathrm{E}-15$ & $3.97 \mathrm{E}-15$ \\
\hline $\mathbf{M}$ & - & 10.5 & 9 \\
\hline $\mathbf{h}_{\mathrm{e}}$ & $\mathrm{mm}$ & \multicolumn{2}{|c|}{1.155} \\
\hline
\end{tabular}

\subsubsection{Homogeneous Model}

A homogeneous FEA model was also analyzed to determine if modeling heterogeneity was necessary. Similar results from both models would suggest that modeling heterogeneity is unnecessary. The properties for the homogeneous model were taken from averaged $\mathrm{S}$ and $\mathrm{M}$ layer properties and are presented in 
Table 5.2. Results similar to the heterogeneous model were generated to compare the physical mechanisms of interest (described below).

\subsection{Physical Mechanisms for Biosynthesis}

\subsubsection{Diffusive Fluid Velocity}

During dynamic UCC of AC, the imbibement and exudation of the interstitial fluid allow nutrients to enter the tissue for biosynthesis and may stimulate cells via fluid induced shear to produce a cellular biosynthetic response. Buschmann et al. [6] conducted experiments that showed stimulation of PG synthesis in areas of the tissue where the fluid velocities exceeded a trigger value of $0.25 \mu \mathrm{m} / \mathrm{s}$. This on-off trigger response was the basis for plotting the magnitude of the relative (diffusive) fluid velocity of the FEA model to determine what regions of the disc would experience PG synthesis during dynamic compression. The study also pointed out that when the tissue was loaded at a frequency of $0.1 \mathrm{~Hz}$ stimulation of PG synthesis occurred only on the periphery of the disc $[6,29]$. The discs were dynamically loaded for 23 hours and allowed to free swell for 16 hours. The experimental test in [37] had a slightly longer growth time and the results are likely better to reflect where the GAGs will be linked to the SM.

ABAQUS outputs the FLVEL variable, which is the effective velocity of the wetting liquid $(f)$ in $\mathrm{mm} / \mathrm{s}$. FLVEL is defined as 


$$
f=s n v_{w}
$$

where $s$ is the saturation, which is set to 1.0 for a fully saturated material, $n$ is the porosity defined in Equation (3.14), and $v_{w}$ is the relative fluid velocity $(\mathrm{mm} / \mathrm{s})$. Some manipulation of Equation (5.1) and inserting the definition of porosity from Equation (3.14) results in the following equation

$$
v_{w}=\frac{f}{\frac{e}{1+e}}
$$

In order to get the desired trigger value in ABAQUS, a new field output variable is defined as RFVEL for relative fluid velocity and is given by

$$
R F V E L=1000 v_{w}=\frac{1000 f}{\frac{e}{1+e}}
$$

where $v_{w}$ is multiplied by 1000 to convert the units to $\mu \mathrm{m} / \mathrm{s}$.

\subsubsection{Maximum Shear Stress}

Another trigger response correlated to biosynthesis is the maximum shear stress subjected to the tissue. Tissue shear loading was used to isolate the effect of biosynthesis due to deformation of the SM versus that of fluid flow due to dynamic loading [16]. Tissue shear causes SM deformation with little volumetric deformation, which causes fluid flow. Jin et al. [16] conducted a study that subjected $\mathrm{AC}$ to a $10 \%$ compressive strain with a superimposed $1 \%$ and $3 \%$ shear 
strains. The results of the study suggested that tissue shear loading provided stimulation of both COL and PG.

Ficklin [10] simulated the simple shear experiment by Jin et al. [16] to determine a maximum shear stress trigger using material properties from the same tissue source used here. In his work, he used the Tresca yield criterion as a measure of maximum shear stress of the tissue and determined the trigger value to be 0.07 MPa. ABAQUS directly produces this Tresca stress field output variable and was used to develop a contour plot of the disc to determine which regions exceeded this trigger value.

\subsubsection{Cell Death}

High compressive strains and their associated stresses have been proven to cause cell death, which leads to AC degradation [44]. Compressive strains above $25 \%$ can cause reduced mechanical properties, denaturation of type II collagen, and a reduction of PG content [46]. Compressive strains exceeding $30 \%$ were found to cause cell death [45]. Compressive stresses of $1.0 \mathrm{MPa}$ have resulted in a significant suppression of PG synthesis, assumed to be due to cell necrosis and tissue damage [13]. Contour plots of the minimum and maximum principal strains and stresses were examined to predict if cell death would occur during the dynamic compression. 


\section{Chapter 6}

\section{Results}

The results presented in this chapter are contour plots generated from ABAQUS. The plots are the $\mathrm{X}-\mathrm{Z}$ plane of the quarter disc model with the center being at radius $0.00 \mathrm{~mm}$ and the outer radial surface at radius $1.25 \mathrm{~mm}$. With the exception of the diffusive fluid velocity results, all data were collected during maximum compression of the disc in the fifth cycle (42.5 s) of the dynamic compression step.

\subsection{Diffusive Fluid Velocity Results}

The data for this plot was collected when the disc experienced the maximum diffusive fluid velocity, which occurred at $41.5 \mathrm{~s}$ into the dynamic compression step. Results from both the heterogeneous and homogeneous model show that the diffusive (relative) fluid velocity exceeds the $0.25 \mu \mathrm{m} / \mathrm{s}$ trigger value on the radial periphery of the quarter disc (Figure 6.1). This trigger was for the stimulation of

GAG synthesis during dynamic UCC [5]. The M-layer displays a higher fluid 
velocity due to its higher permeability. The highest fluid velocity occurs on the M-layer side of the interface and exceeds $1.25 \mu \mathrm{m} / \mathrm{s}$.

(A)
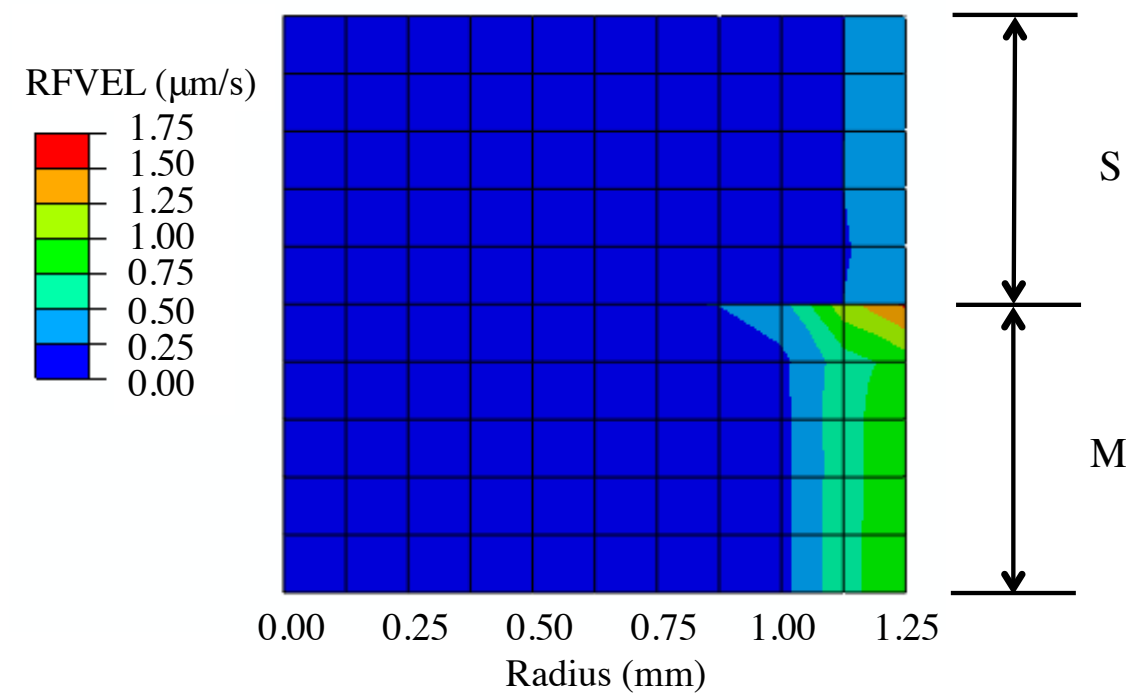

M

(B)
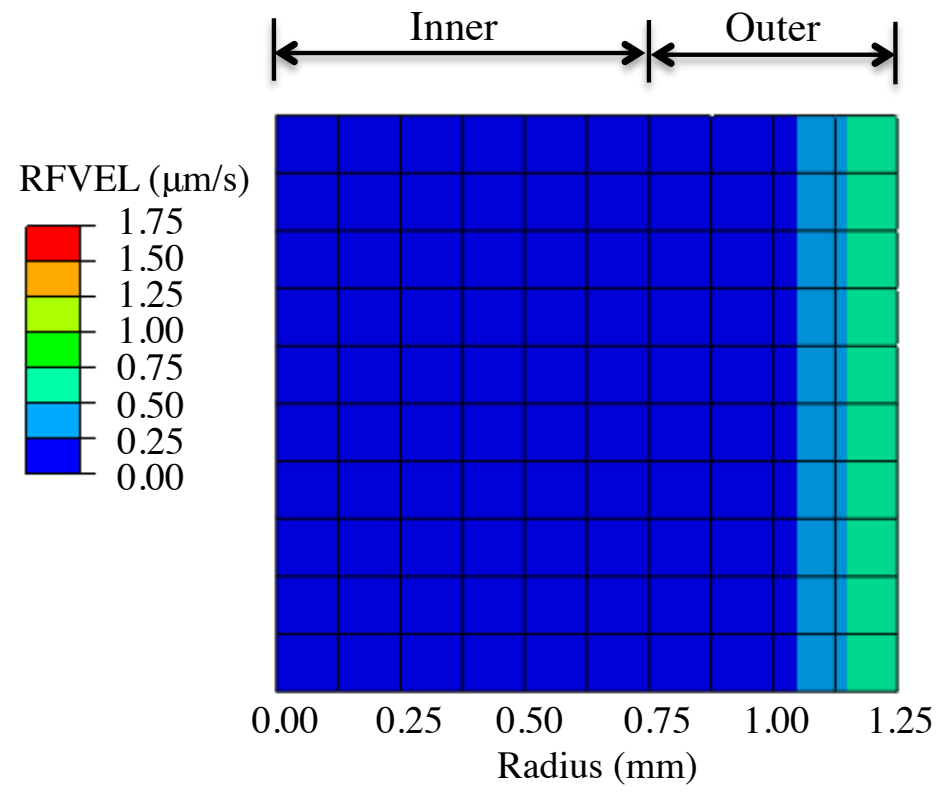

Figure 6.1. Contour plots of the relative fluid velocity magnitude for the heterogeneous $^{4}(\mathrm{~A})$ and homogeneous (B) models. Results show that high relative fluid velocities exist at the radial periphery of the disc for both models.

${ }^{4}$ A discussion about the discontinuity of the fluid velocity between the two layers in Figure 6.1 (A) is provided in the Appendix. 


\subsection{Tresca Stress Results}

The Tresca stress is predicted to exceed the 0.07 MPa trigger, which Ficklin [10] developed, in a majority of the disc in Figure 6.2 except at the radial periphery. This trigger was established from the dynamic shear experiments that stimulated COL and GAG synthesis in [16]. The heterogeneous and homogeneous models present similar results except for the heterogeneous model, lower values of Tresca stress exist on the S-layer side of the interface. The highest value of the Tresca stress is located around the $0.75 \mathrm{~mm}$ radial region and is slightly higher for the heterogeneous model at $0.090 \mathrm{MPa}$. 
(A)

$\mathrm{S}$, Tresca (MPa)
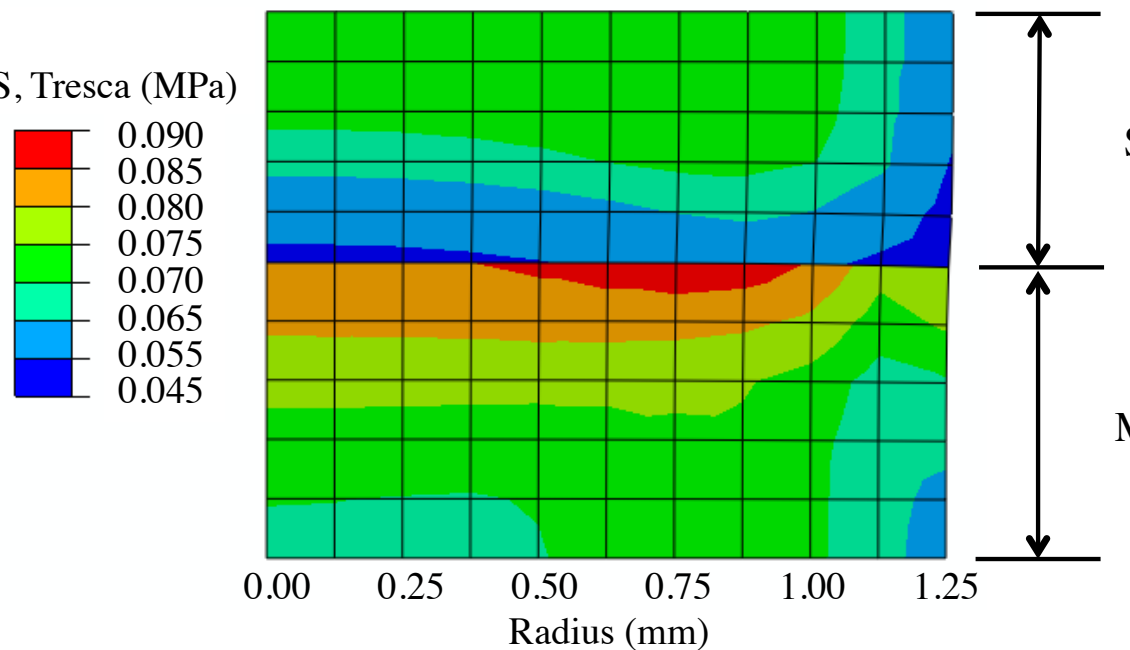

S

M

(B)

$\mathrm{S}$, Tresca (MPa)
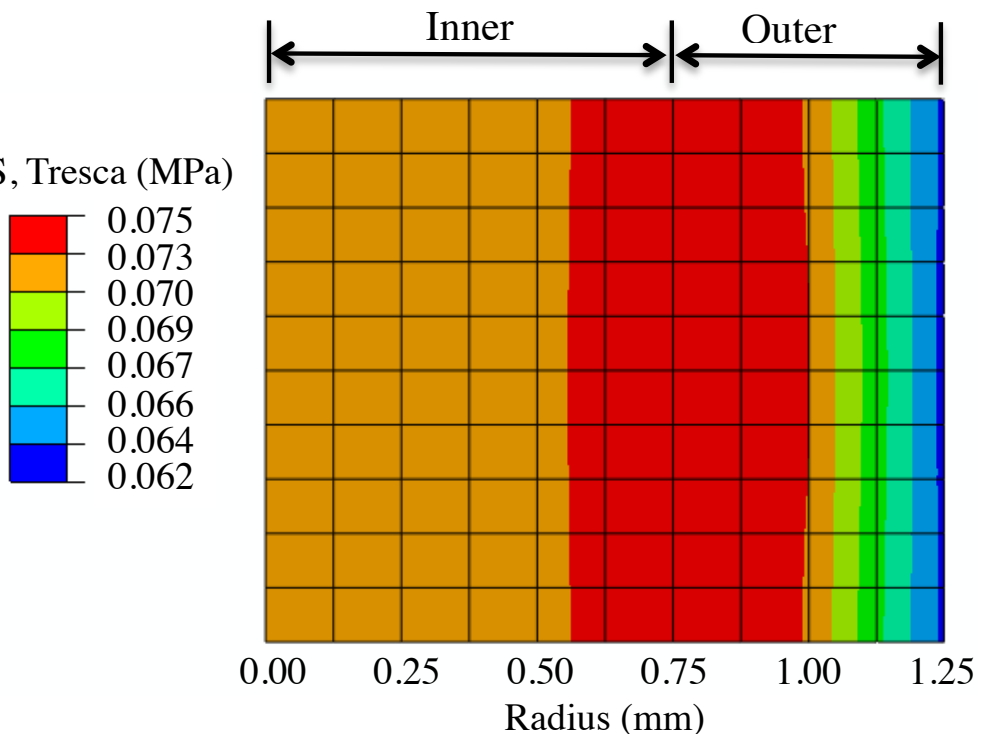

Figure 6.2. Contour plots of the Tresca stress for the heterogeneous (A) and homogeneous (B) models. The Tresca stress exceeded the $0.07 \mathrm{MPa}$ trigger value at a majority of the disc except at the radial periphery. 


\subsection{Radial Strain Results}

Due to the tensile load carrying nature of COL fibers, a trigger value for radial strain could be proposed for COL synthesis, although there is no research to support a strain trigger value. If a strain trigger were adopted, the results in Figure 6.3 suggest that stimulation of COL synthesis would be nearly uniform except near the periphery. This would suggest that the biochemical data that shows homogeneous COL contents of the inner core and outer ring resulted from a nearly homogeneous strain trigger. However, a comparison of the D0 and S10+D COL contents determined that COL synthesis did not occur during dynamic UCC. 
(A)

EE11,

Radial Strain

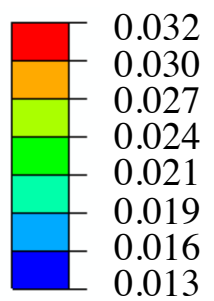
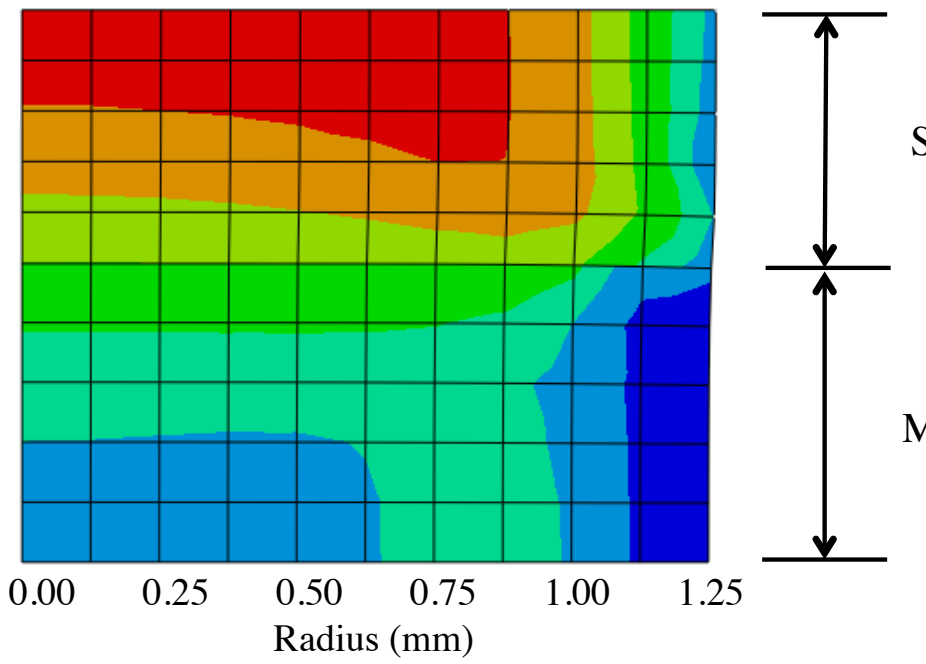

EE11,

Radial Strain
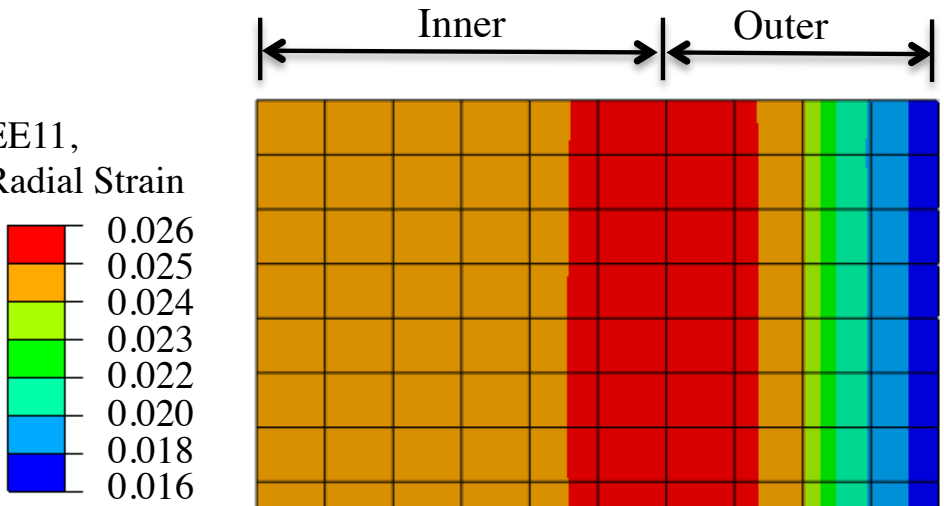

(B)

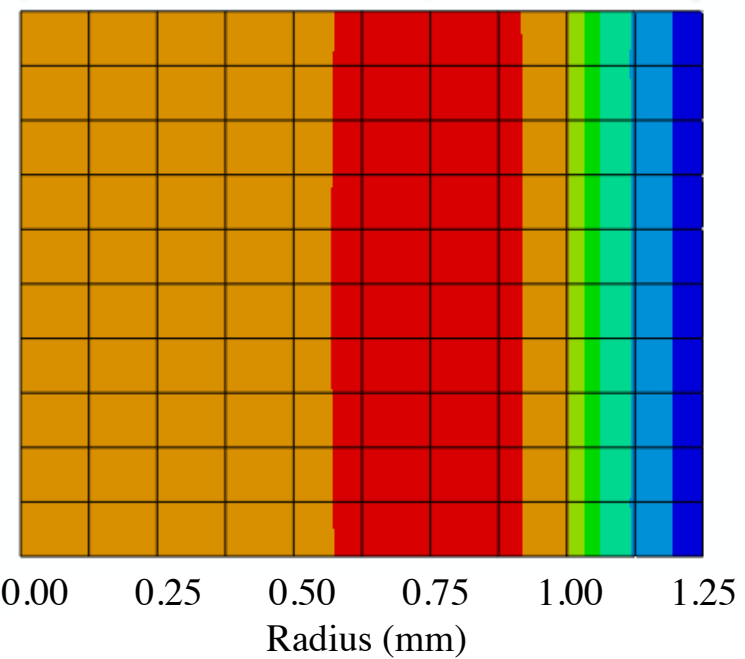

Figure 6.3. Contour plots of the radial strain for the heterogeneous (A) and homogeneous (B) models. It was suggested that radial strain could stimulate COL synthesis and the results are presented here for discussion. 


\subsection{Cell Death Results}

The contour plots of the maximum and minimum principal stresses and strains are presented to predict if cell necrosis would occur during the analysis. As previously mentioned in 5.3.3 Cell Death, cell necrosis and tissue damage are expected to occur when the tissue experiences compressive strains exceeding $25 \%$ [46] and compressive stresses at 1.0 MPa [13]. The contour plots below show that none of these models reached a compressive stress of 1.0 MPa. It is important to note that this is not a trigger value, and that cell necrosis could occur at compressive stress values lower than 1.0 MPa. 


\subsubsection{Minimum Principal Stress Results}

(A)
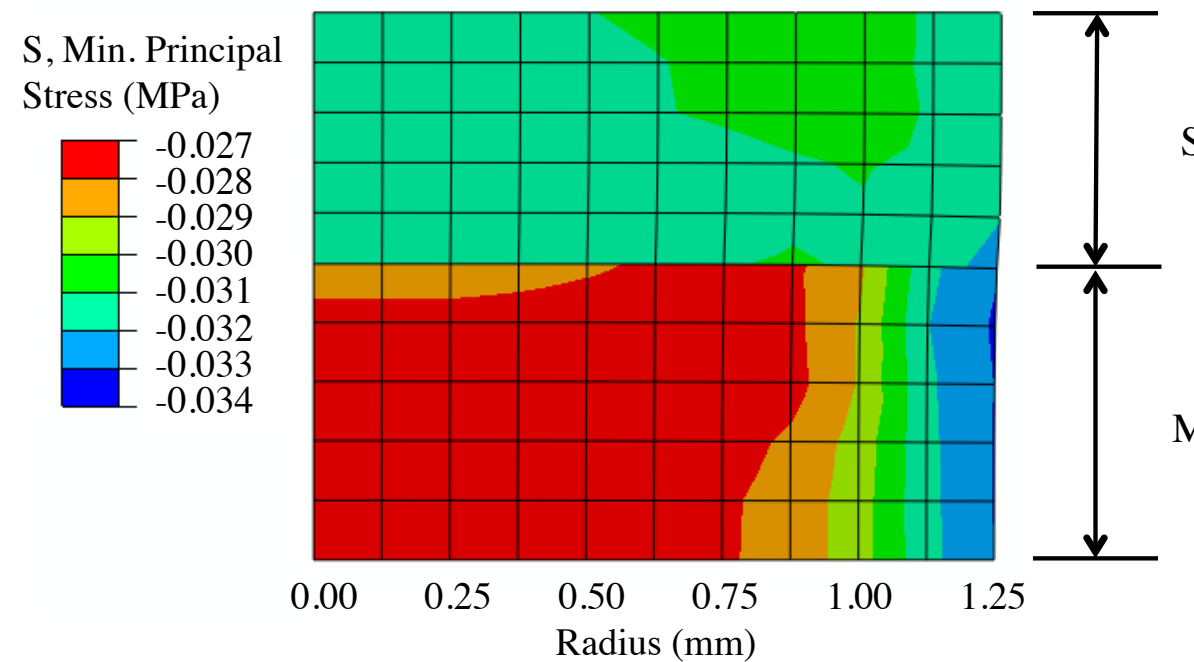

$\mathrm{S}$

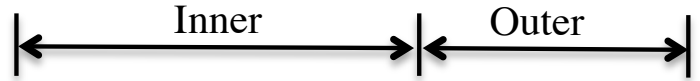

S, Min. Principal Stress (MPa)

\begin{tabular}{|l}
-0.031 \\
-0.031 \\
-0.032 \\
-0.032 \\
-0.033 \\
-0.033 \\
-0.033 \\
-0.034 \\
\hline
\end{tabular}

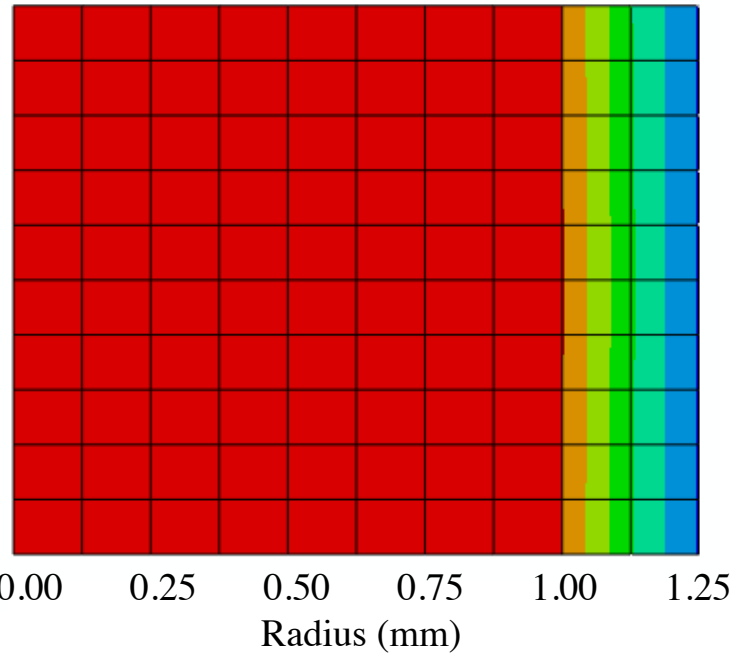

Figure 6.4. Contour plots of the minimum principal stress for the heterogeneous (A) and homogeneous (B) models. 


\subsubsection{Minimum Principal Strain Results}

(A)
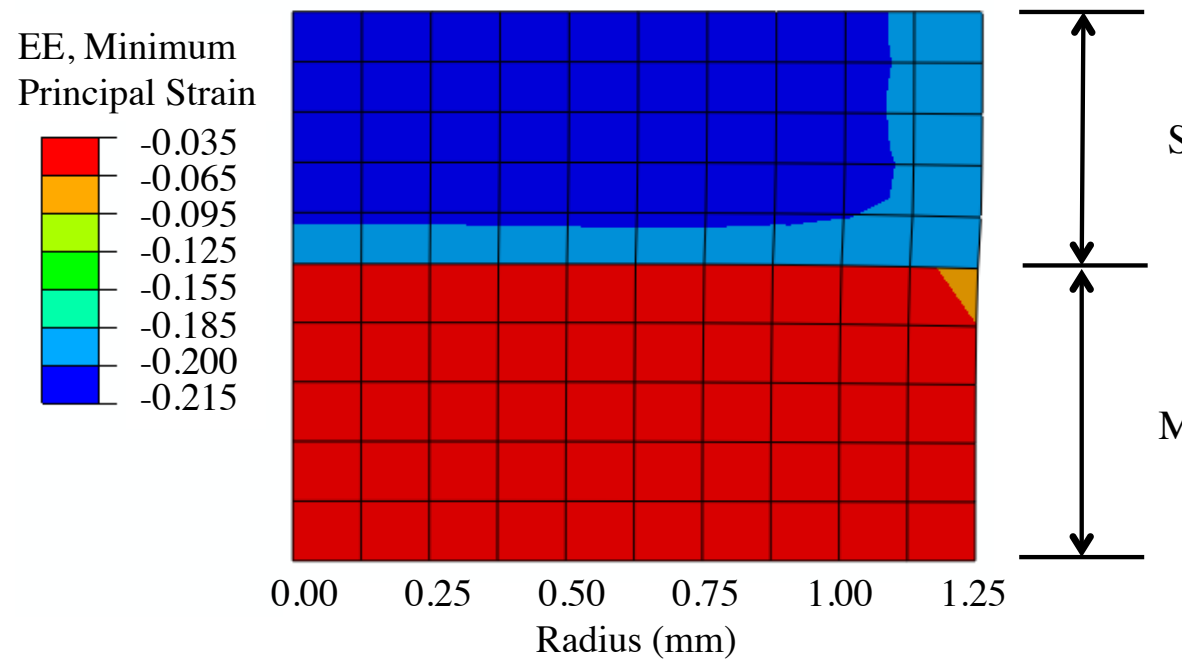

(B)

EE, Minimum Principal Strain
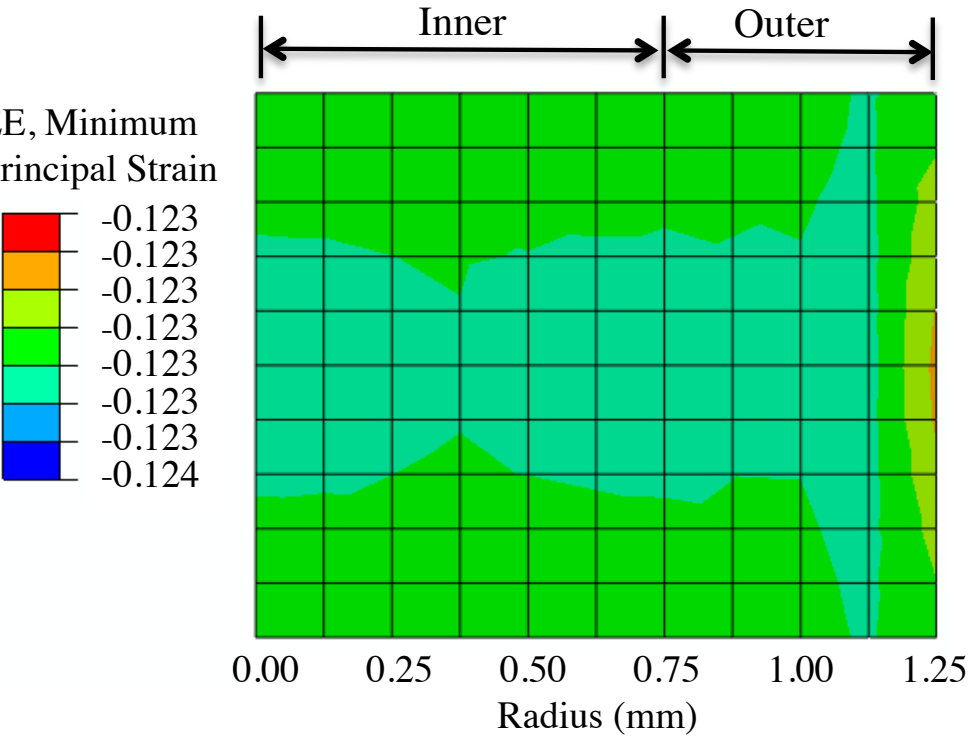

Figure 6.5. Contour plots of the minimum principal strain for the heterogeneous (A) and homogeneous (B) models.

The heterogeneous model in Figure 6.5 (A) shows the S-layer absorbing much of the compressive strains, which reach a maximum of $21.5 \%$. Although this does not exceed the $25 \%$ compressive strain that is expected to result in cell death [46], the material properties for this model are only at $50 \%$ of the actual experimental 
values due to convergence issues. If a fully-fledged heterogeneous model was functional, the S-layer may reach higher levels of strain and consequently suggest that cell death and AC degradation may occur.

\subsubsection{Maximum Principal Stress Results}

(A)

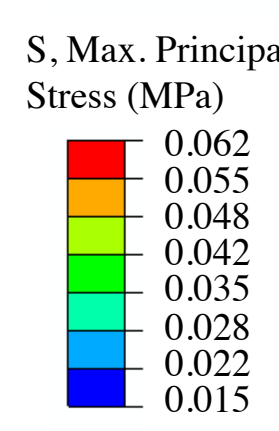

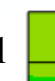

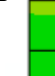
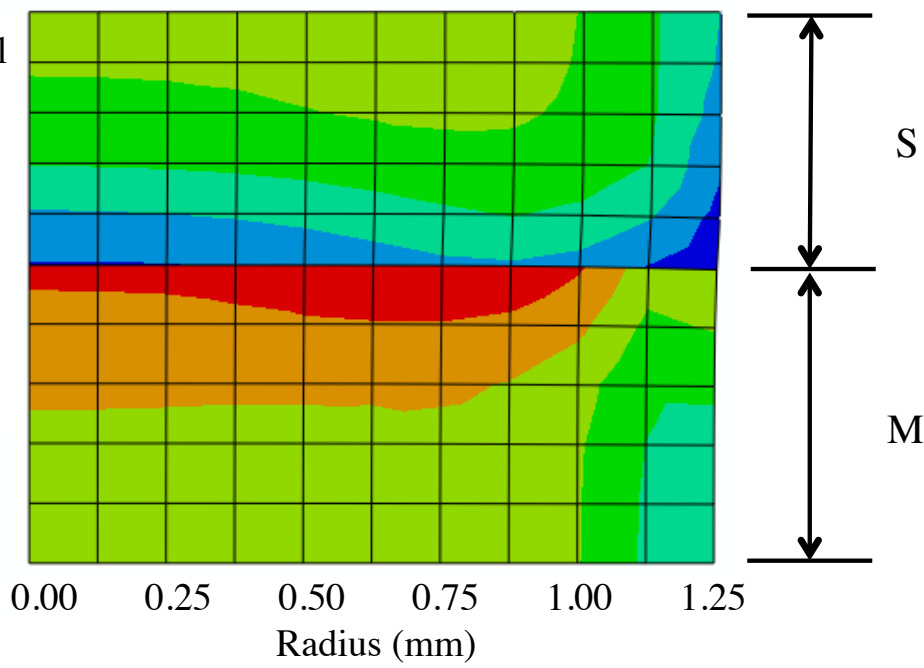

(B)

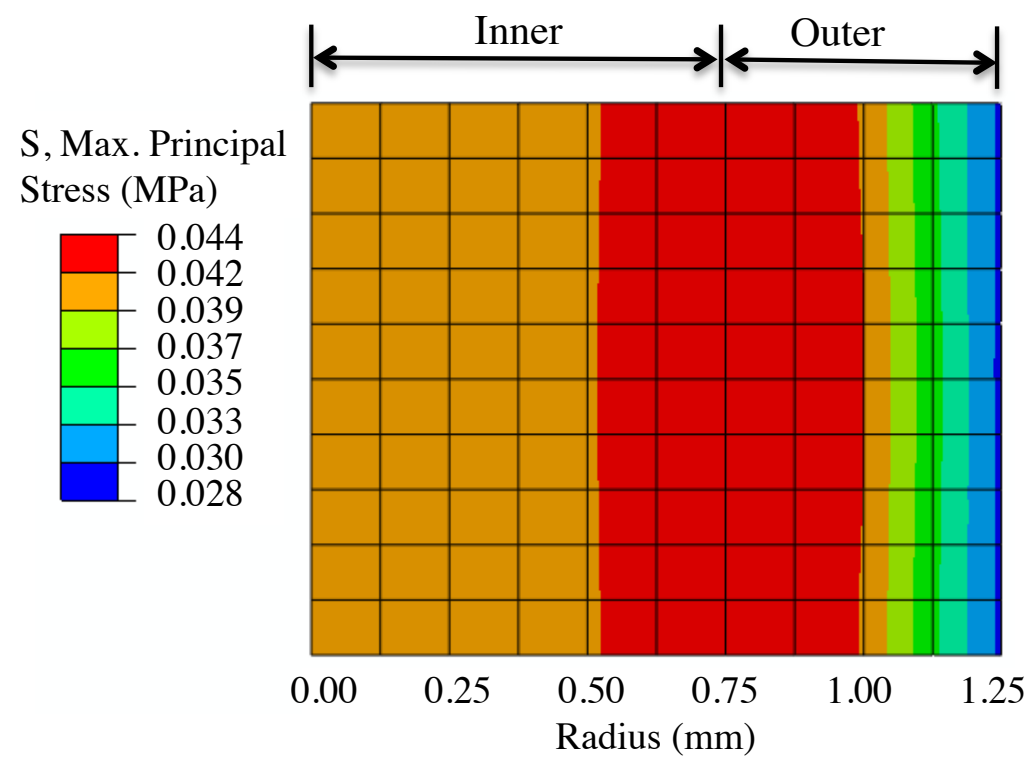

Figure 6.6. Contour plots of the maximum principal stress for the heterogeneous (A) and homogeneous (B) models. 


\subsubsection{Maximum Principal Strain Results}

(A)
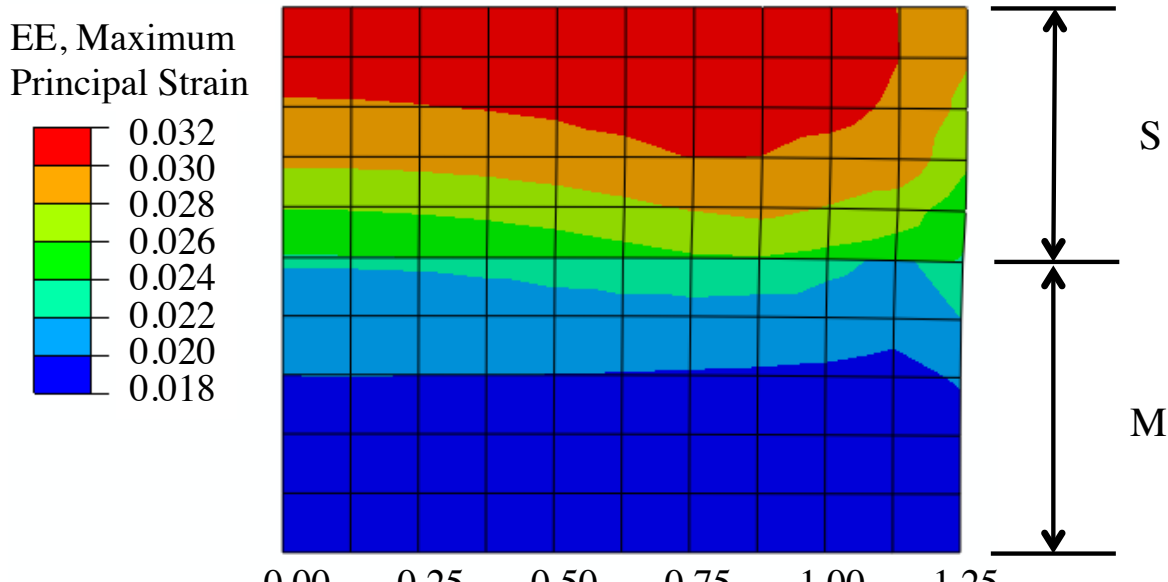

EE, Maximum Principal Strain

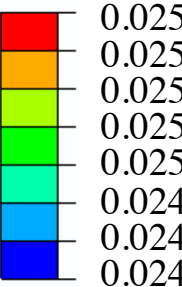

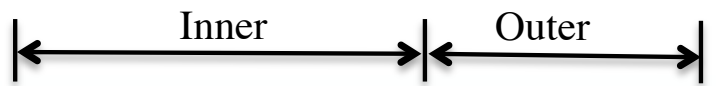

(B)

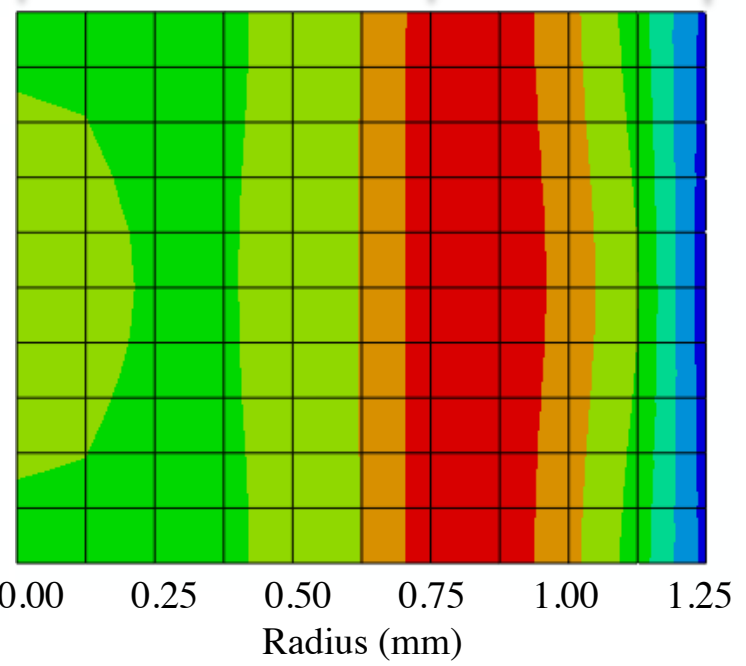

Figure 6.7. Contour plots of the maximum principal strain for the heterogeneous (A) and homogeneous (B) models.

Similar to radial strain, maximum principal strain could stimulate COL synthesis due to the tensile load carrying nature of COL. The results in Figure 6.7 suggest that there would be no radial difference in COL content, as expected from the 
experimental data presented in the previous chapter. However, a comparison of D0 and S10+D COL contents determined that COL synthesis did not occur. This may indicate that these strains aren't high enough to stimulate COL synthesis. 


\section{Chapter 7}

\section{Discussion}

The biochemical results [37] provided did not include data from D0 specimens to determine whether or not stimulation of COL or GAG synthesis occurred after dynamic UCC. However, the D0 biochemical data from [34] were used as a reference to determine if synthesis occurred. This is the same source that provided data for the $\mathrm{S}$ and $\mathrm{M}$ layer material properties used in this analysis. D0 specimens from [37] are available, but biochemical tests will be conducted at a later time.

\subsection{GAG Synthesis}

The biochemical results in Figure $\mathbf{5 . 1}$ provide evidence that dynamic compression ( $\pm 2 \%$ strain) of the tissue at $0.1 \mathrm{~Hz}$ causes a higher stimulation of GAG synthesis in the outer ring or at the radial periphery in comparison to D0 samples. The only physical mechanism of those examined in this computational analysis that produces a similar periphery result is the contour plot of the diffusive (relative) velocity. If the $0.25 \mu \mathrm{m} / \mathrm{s}$ trigger from Buschmann et al. [6] were adopted, biosynthesis would occur within the outer ring with a radius from 1.00 to 
$1.25 \mathrm{~mm}$. This supports the hypothesis that the fluid velocity in the tissue stimulates GAG synthesis when the velocity exceeds the trigger value. These results may support the use of the fluid velocity trigger for GAG synthesis in future FEA growth and remodeling analyses and may also suggest a different radial punch (from 1.00 to $1.25 \mathrm{~mm}$ ) when conducting future experimental dynamic UCC tests.

The binary contour plot of Figure 7.1 displays regions of the model that surpass the $0.07 \mathrm{MPa}$ Tresca stress trigger. A majority of the plot exceeds this trigger, predominantly in the inner core, but also within the outer ring. The existence of the exceeded trigger in both regions suggests that the Tresca stress trigger is not the predominant physical mechanism that stimulates GAG synthesis. However, in comparison to the D0 data, S10+D GAG content was higher in both the inner core and outer ring. This suggests that the use of the Tresca stress trigger could provide minor stimulation of GAG synthesis in future FEA growth and remodeling analyses.

\subsection{COL Synthesis}

The biochemical results for COL content are homogeneous after the dynamic loading due to the inner core and outer ring not being statistically different. This would suggest that fluid velocity is not the dominant physical mechanism that stimulates COL synthesis because of the heterogeneity (high velocities at the 
periphery) seen in the results of the computational study. Although a majority of the contour plot experienced a Tresca stress value that surpassed the trigger (Figure 7.1), which would suggest that shear stress stimulation is homogeneous and would support the findings of the biochemical results after dynamic loading, a comparison of the D0 and S10+D results indicated that there was not a significant difference between the COL contents for the inner core, suggesting that synthesis did not occur in the inner core. The statistical analysis also indicated that the COL content for the S10+D outer ring was less than the D0 COL content. This would suggest denaturation of COL in the outer periphery. 
$\mathrm{S}$, Tresca (MPa)

0.090

0.070

0.000

(A)
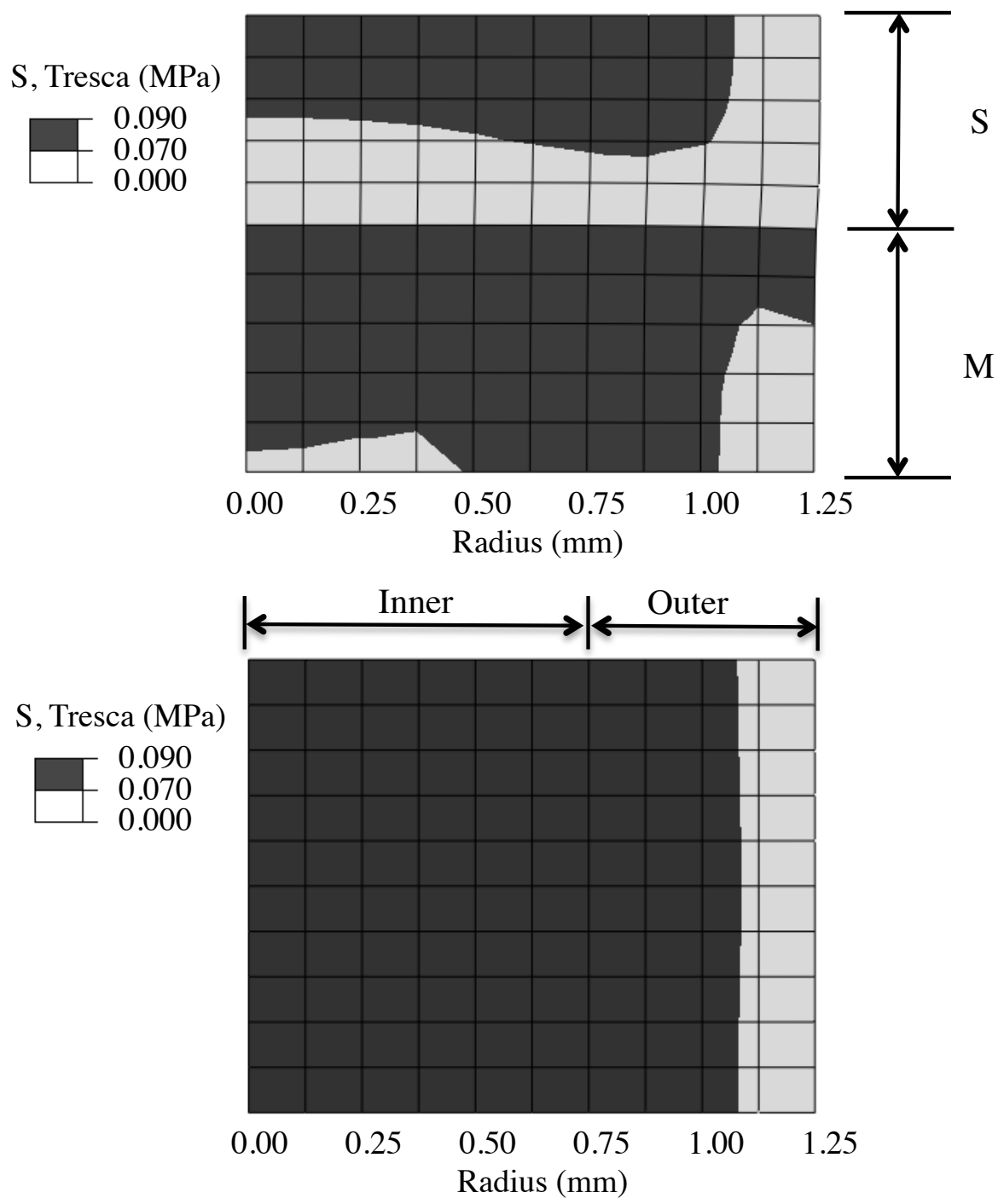

(B)

Figure 7.1. Binary contour plots of the Tresca stress for the heterogeneous (A) and homogeneous (B) models. The darker regions show where the Tresca stress exceeded the $0.07 \mathrm{MPa}$ trigger value.

\subsection{Feasibility of Heterogeneity}

The computational analysis times for the heterogeneous and homogeneous analyses are presented in Table 7.1. The complex heterogeneous and homogeneous models took a total of 77.5 and 56 hours, respectively. Both 
analyses ran on the same computer at separate times to determine the computational time. The technical specifications of the computer are listed in the Appendix. Although ABAQUS was not able to converge during an analysis with full-fledged S and M-layer properties, an increase in computational power may aid in continuing to investigate ways to get the full-fledged heterogeneous model operational. Suggestions for future work are presented in the Appendix.

Table 7.1. Computational analysis times for the heterogeneous and homogeneous models. Durations for each step are presented to compare (hh:mm:ss).

\begin{tabular}{|l|c|c|c|c|c|}
\cline { 2 - 6 } \multicolumn{1}{c|}{} & Equilibrium & $\begin{array}{c}\text { Initial } \\
\text { Compression }\end{array}$ & $\begin{array}{c}\text { Stress } \\
\text { Relaxation }\end{array}$ & $\begin{array}{c}\text { Dynamic } \\
\text { Compression }\end{array}$ & Total \\
\hline Heterogeneous & $0: 10: 03$ & $6: 24: 39$ & $1: 52: 09$ & $67: 07: 51$ & $77: 29: 31$ \\
\hline Homogeneous & $0: 10: 19$ & $3: 47: 04$ & $2: 31: 26$ & $49: 25: 19$ & $55: 54: 08$ \\
\hline
\end{tabular}

Convergence problems could also be due to the high strains subjected to the Slayer. As evident in Figure 6.5 (A), the largest minimum principal strain (compressive strain) values exist in the S-layer. This indicates that the S-layer absorbs most of the compressive strain being applied to the entire tissue. High strains cause compaction of the pores, resulting in a very low permeability and very high pressure within the tissue causing convergence problems in the analysis [10][22]. It is important to note that an elastic analysis with full-fledged $\mathrm{S}$ and $\mathrm{M}$ layer elastic $(\mathrm{SM})$ properties converged, which indicates that the poroelastic analysis with permeability is causing convergence issues. The discontinuity in the void ratios and consequently the permeability could be the factor in causing convergence problems in ABAQUS. 


\section{List of References}

[1] Ateshian, G. A., Wang, H., \& Lai, W. M. (1998). The role of interstitial fluid pressurization and surface porosities on the boundary friction of articular cartilage. Journal of Tribology, 120, 241-248.

[2] Ateshian, G. A., Warden, W. H., Kim, J. J., Grelsamer, R. P., \& Mow, V. C. (1997). Finite deformation biphasic material properties of bovine articular cartilage from confined compression experiments. Journal of Biomechanics, 30, 1157-1164.

[3] Atkin, R. J., \& Crane, R. E. (1976). Continuum theories of mixtures: basic theory and historical development. Q J Mechanics Appl Math, 29 (2), 209244.

[4] Buckwlater, J. A., \& Mankin, H. J. (1998). Articular cartilage repair and transplantation. Arthritis \& Rheumatism, 41 (8), 1331-1342.

[5] Buschmann, M. D., \& Grodzinsky, A. J. (1995). A molecular model of proteoglycan-associated electrostatic forces in cartilage mechanics. Journal of Biomechanical Engineering, 117, 179-192.

[6] Buschmann, M. D., Kim, Y.-J., Wong, M., Frank, E., Hunziker, E. B., \& Grodzinsky, A. J. (1999). Stimulation of aggrecan synthesis in cartilage explants by cyclic loading is localize to regions of high interstitial fluid flow. Archives of Biochemistry and Biophysics, 366 (1), 1-7.

[7] Chen, A. C., Bae, W. C., Schinagl, R. M., \& Sah, R. L. (2001). Depth- and strain-dependent mechanical and electromechanical properties of fullthickness bovine articular cartilage in confined compression. Journal of Biomechanics, 34, 1-12.

[8] Cheng, Y., Hootman, J., Murphy, L., Langmaid, G., \& Helmick, C. (2010, October 8). Prevalence of Doctor-Diagnossed Arthritis and ArthritisAttributable Activity Limitation. CDC: Morbidity and Mortality Weekly Report, 59 (39), pp. 1261-1265.

[9] Dassault Systemes. (2007). ABAQUS 6.7 Analysis User's Manual.

[10] Ficklin, T. P. (2007). Application of a cartilage growth finite element model: Simulation of steady-state fluid permeation experiments. California Polytechnic State University, San Luis Obispo. 
[11] Ficklin, T. P., Davol, A., \& Klisch, S. M. (2009). Simulating the growth of articular cartilage explants in a permeation bioreactor to aid in experimental protocol design. Journal of Biomechanical Engineering, 131,1-11.

[12] Guilak, F., \& Mow, V. C. (2000). The mechanical environment of the chondrocyte: a biphasic finite element model of cell-matrix interactions in articular cartilage. Journal of Biomechanics, 33, 1663-1673.

[13] Guilak, F., Meyer, B. C., Ratcliffe, A., \& Mow, V. C. (1994). The effects of matrix compression on proteoglycan metabolism in articular cartilage explants. Osteoarthritis and Cartilage, 2, 91-101.

[14] Holmes, M. H., \& Mow, V. C. (1990). The Nonlinear Characteristics of Soft Gels and Hudrated Connective Tissues in Ultrafiltration. Journal of Biomechanics, 23, 1145-1156.

[15] Insall, J. N., Ranawat, C. S., Aglietti, P., \& Shine, J. (1976). A comparison of four models of total knee-replacement prostheses. The Journal of Bone and Joint Surgery, 58, 754-765.

[16] Jin, M., Frank, E. H., Quinn, T. M., Hunziker, E. B., \& Grodzinsky, A. J. (2001). Tissue shear deformation stimulates proteoglycan an proetin biosynthesis in bovine cartilage explants. Archives of Biochemistry and Biophysics, 395 (1), 41-48.

[17] Kim, Y.-J., Sah, R. L., Grodzinsky, A. J., Plaas, A. H., \& Sandy, J. D. (1994). Mechanical regulation of cartilage biosynthetic behavior: Physical stimuli. Archives of Biochemistry and Biophysics , 311 (1), 1-12.

[18] Klein, T. J., Chaudhry, M., Bae, W. C., \& Sah, R. L. (2007). Depthdependent biomechanical and biochemical properties of fetal, newborn, and tissue-engineered articular cartilage. Journal of Biomechanics, 40, $182-190$.

[19] Klisch, S. M., Asanbaeva, A., Oungoulian, S. R., Masuda, K., Thonar, E. J., Davol, A., \& Sah, R. L. (2008). A cartilage growth mixture model with collagen remodeling: validation protocols. Journal of Biomechanical Engineering, 130, 031006.

[20] Klisch, S. M., \& Lotz, J. C. (2000). A Special Theory of Biphasic Mixtures and Experimental Results for Human Annulus Fibrosus Tested in Confined Compression. Journal of Biomechanical Engineering, 122, 180-188.

[21] Kuettner, K. E. (1992). Biochemistry of articular cartilage in health and disease. Clinical Biochemistry, 25 (3), 155-163. 
[22] Lai, W. M., \& Mow, V. C. (1980). Drag-induced compression of articular cartilage during a permeation experiment. Biorheology, 17, 111-123.

[23] Li, L. P., Buschmann, M. D., \& Shirazi-Adl, A. (2000). A fibril reinforced nonhomogeneous poroelastic model for articular cartilage: inhomogeneous response in unconfined compression. Journal of Biomechanics, 33, 1533-1541.

[24] Morel, V., \& Quinn, T. M. (2004). Cartilage injury by ramp compression near the gel diffusion rate. Journal of Orthopaedic Research, 22, 145-151.

[25] Mow, V. C., Kuei, S. C., Lai, W. M., \& Armstrong, C. G. (1980). Biphasic creep and stress relaxation of articular cartilage in compression: Theory and experiments. Journal of Biomechanical Engineering, 102, 7384.

[26] Pendergast, P., van Driel, W., \& Kuiper, J.-H. (1997). A comparison of finite element codes for the solution of biphasic poroelastic problems. Journal of Engineering in Medicine, 210, 131-136.

[27] Quinn, T. M., Allen, R. G., Schalet, B. J., Perumbuli, P., \& Hunziker, E. B. (2001). Matrix and cell injury due to sub-impact loading of adult bovine articular cartilage explants: effects of strain rate and peak stress. Journal of Orthopaedic Research, 19, 242-249.

[28] Raghunathan, S., Evans, D., \& Sparks, J. L. (2010). Poroviscoelastic modeling of liver biomechnical resonpse in unconfined compression. Annals of Biomedical Engineering, 38 (5), 1789-1800.

[29] Sah, R. L.-Y., Kim, Y.-J., Doong, J.-Y. H., Grodzinsky, A. J., Plaas, A. H., \& Sandy, J. D. (1989). Biosynthetic response of cartilage explants to dynamic compression. Journal of Orthopaedic Research, 7 (5), 619-636.

[30] Shirazi, R., Vena, P., Sah, R. L., \& Klisch, S. M. (2011). Modeling collagen fibrils using a continuous volume fraction distribution function for use. Mathematics and Mechanics of Solids, in press.

[31] Spencer, A. J. (1980). Continuum Mechanics. Mineola, NY: Dover Publications, Inc.

[32] Stender, M. E. (2011). Predicting articular cartilage constituent material properties following in vitro growth using a proteoglycan-collagen mixture model. California Polytechnic State University, San Luis Obispo. 
[33] Waldman, S. D., Spiteri, C. G., Grynpas, M. D., Pilliar, R. M., Hong, J., \& Kandel, R. A. (2003). Effect of biomechanical conditioning on cartilaginous tissue formation in vitro. The Journal of Bone and Joint Surgery, 85, 101-105.

[34] Williams, G. M., Dills, K. J., Flores, C. R., Stender, M. E., Stewart, K. M., Nelson, L. M., et al. (2010). Differential regulation of immature articular cartilage compressive moduli and Poisson's ratios by in vitro stimulation with IGF-1 and TGF-B1. Journal of Biomechanics, 43, 2501-2507.

[35] Williamson, A. K., Chen, A. C., \& Sah, R. L. (2001). Compressive properties and function-composition relationships of developing bovine articular cartilage. Journal of Orthopaedic Research, 19, 1113-1121.

[36] Wu, J. Z., Herzon, W., \& Epstein, M. (1998). Evaluation of the finite element software ABAQUS for biomechanical modelling of biphasic tissues. Journal of Biomechanics, 31, 165-169.

[37] Yamauchi, K. (2010). [Project \#3 Biochem and mechanical testing results]. Unpublished raw data.

[38] Ouyang, F. (2005). ABAQUS Implementation of Creep Failure in Polymer Matrix Composites with Transverse Isotropy. The University of Akron, Akron, $\mathrm{OH}$.

[39] Stender, M. E., Balcom, N., Berg-Johansen, B., Dills, K. J., Dyk, D., Sah, R. L., Klisch, S. M., \& Hazelwood, S. J. (2011). Differential regulation of articular cartilagetTensile properties by IGF-1 and TGF-betal during in vitro growth. Submitted to: International Conference on the Mechanics of Biomaterials and Tissues.

[40] Klisch, Stephen M. (2008). Cal Poly Cartilage Biomechanics Group. Retrieved from http://www.calpoly.edu/ sklisch/biogroup/index.htm

[41] Healy, W. L., Iorio, R., Ko, J., Appleby, D., \& Lemos, D. W. (2002). Impact of cost reduction programs on short-term patient outcome and hospital cost of total knee arthroplasty. The Journal of Bone and Joint Surgery, 84-A (3), 348-353.

[42] Beavers, G. S. \& Joseph, D. D. (1967). Boundary condition at a naturally permeable wall. Journal of Fluid Mechanics, 30 (1), 197-207.

[43] Jager, W. \& Mikelic, A. (2000). On the interface boundary condition of Beavers, Joseph, and Saffman. SIAM Jounal on Applied Mathematics, 60 (4), 1111-1127. 
[44] Bae, W. C., Lewis, C. W., Levenston, M. E., \& Sah, R. L. (2006). Indentation testing of human articular cartilage: Effects of probe tip geometry and indentation depth on intra-tissue strain. Journal of Biomechanics, 39, 1039-1047.

[45] D'Lima, D. D., Hashimoto, S., Chen, P. C., Lotz, M. K., \& Colwell Jr., C. W. (2001). Cartilage injury induces chondrocyte apoptosis. Journal of Bone and Joint Surgery-American, 83A (Suppl. 2), 19-21.

[46] Thibault, M., Poole, A. R., \& Buschmann, M. D. (2002). Cyclic compression of cartilage/bone explants in vitro leads to physcial weakening, mechanical breakdown of collagen and release of matrix fragments. Journal of Orthopaedic Research, 20, 1265-1273.

[47] Thomas, G. C., Asanbaeva, A., Vena, P., Sah, R. L., \& Klisch, S. M. (2009). A nonlinear constituent based viscoelastic model for articular cartilage and analysis of tissue remodeling due to altered glycosaminoglycan-collagen interactions. Journal of Biomechanical Engineering, 131, 101002.

[48] Wu, Lan. (2008). In-vivo Cartilage Contact Biomechanics: An experimental and computational investigation of human ankle joint complex. Massachusetts Institute of Technology, Massachusetts. 


\section{Appendix}

\section{A.1 Discontinuity of Fluid Velocity}

The discontinuity of the relative fluid velocity in Figure 6.1 (A) between the S and $\mathrm{M}$ layers is due to the discontinuity in the void ratios. As the fluid flows in and out of the tissue during compression, the velocity tangential to the interface may be discontinuous [42]. Figure A.1 (B) shows the discontinuity of the relative fluid velocity in the x-direction, which is tangential to the interface. However, the velocity normal to the interface must be continuous [42, 43]. Figure A.1 (A) shows the continuity of the relative fluid velocity in the z-direction, which is normal to the interface. 

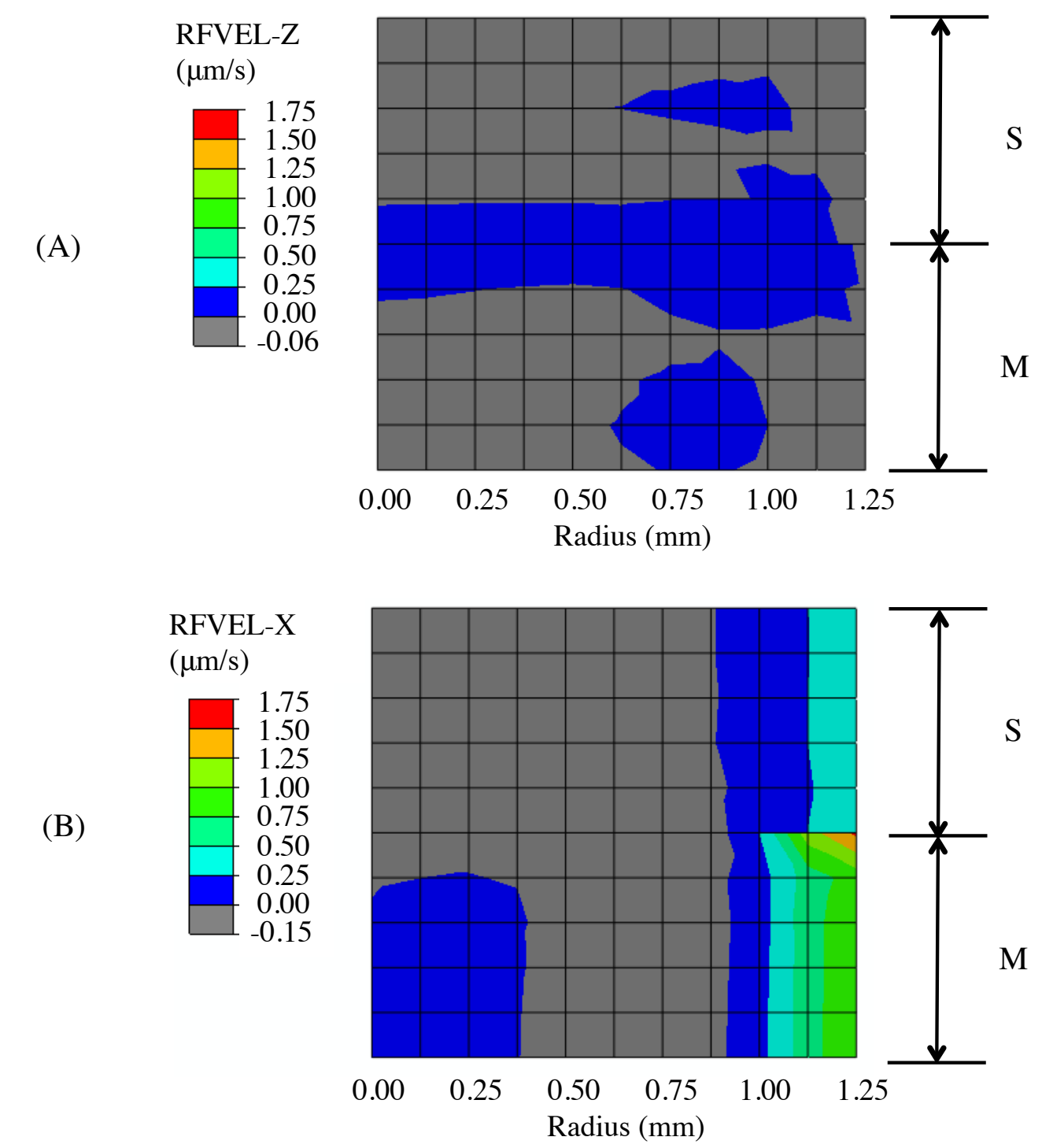

Figure A.1. The relative fluid velocity in the z-direction (A) shows a continuous normal velocity across the discontinuous interface of the two layers. The velocity in the $\mathrm{X}$-direction (B) shows a discontinuous tangential velocity across the interface. 


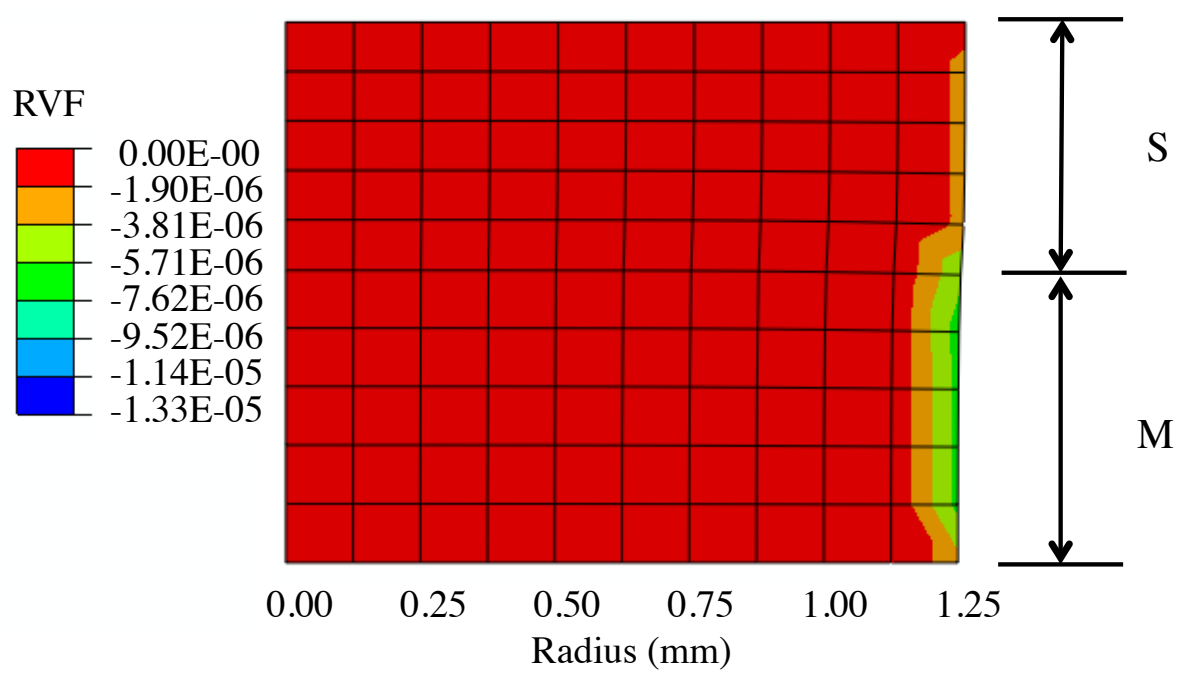

Figure A.2. Reaction fluid volume flux (RVF) due to the pore pressure boundary condition on the radial surface.

The RVF variable is the reaction fluid volume flux due to the pore pressure boundary condition on the radial surface. It is defined as the rate at which the volume of fluid is entering or exiting the model through a node to maintain the pressure BC. A negative value indicates that fluid is exiting the model. Figure A.2 is provided here to show that the continuity of the volume of fluid is maintained between the interface of the $\mathrm{S}$ and $\mathrm{M}$ layers of the heterogeneous model. 


\section{A.2 Suggestions for Future Work}

Because the separation of properties in this model was set at only $50 \%$ from the experimental values, here are suggestions to help with convergence with fullfledged properties. See list of previous failed attempts at modeling for convergence.

1. Create a two-layer model with full-fledged $\mathrm{S}$ and $\mathrm{M}$ elastic (SM) properties with one permeability function based on average properties. Assign full-fledged initial void ratios to each layer. If this doesn't converge, assign one initial void ratio for the entire disc.

2. Create a model with full-fledged $\mathbf{S}$ and $\mathbf{M}$ material properties and assign a finer mesh at the interface to help with convergence and the discontinuity of properties. This should reduce analysis time by having less nodes and elements due to having a coarser mesh away from the interface.

3. Test to see if convergence problems are due to the change in properties or the properties of the S-layer. If the S-layer is absorbing much of the compressive strain and causing convergence issues, the characteristics of the layer could make it too "soft." Properties corresponding to a layer with $\mathrm{E}_{\mathrm{f}}=320 \mathrm{MPa}$ could be too low. 


\section{A.3 Previous Failed Attempts at Convergence}

Many different analyses were attempted at modeling a poroelastic disc for convergence. However, a full-fledged heterogeneous disc based on experimental properties was not successfully created. This list of failed attempts is provided so that future work does not attempt the same analyses. The first three listed attempts were to cause the analysis to pass just the equilibrium step (allows tissue to swell), which was remedied by forcing the step to one increment. The remaining list diverged in the dynamic compression step just after maximum compression (after five seconds).

1. A parameter study was conducted on the SM material properties for the $S$ and M layer to see if they affected convergence. The MAT shear modulus $(\mu)$ was increased by an order of magnitude to 1.0. Although the model converged, the material expanded less than expected during the equilibrium step.

2. Parameter studies were conducted by varying the relative amounts of the reference configuration GAG density $\left(\rho_{o}^{P G}\right)$ and the COL volume fraction $\left(\Phi_{f}\right)$ between the S and M layers. The model only converged when the two layers contained the same $\rho_{o}^{P G}$ value. A parameter study was conducted on this variable, but the model diverged once the separation in the values reached only a $5 \%$ difference. The $\rho_{o}^{P G}$ variable affects the swelling stress of PG (see Equation (3.29)). 
3. A parameter study was also conducted on the $\alpha_{2}$ value, which affects the exponent in the swelling stress of PG (see Equation (3.29)). The value was adjusted from 1.0 (i.e. a linear function) to its original value of 2.5. The highest value for $\alpha_{2}$ while maintaining convergence was 2.48, however, the material expanded less than expected due to the variable's effect on the swelling stress of PG.

4. A multi-layered (more than two) model was also attempted where the $\mathrm{S}$ and $\mathrm{M}$ layer properties (elastic and poroelastic) were varied through the depth of the tissue. To determine the maximum separation of properties between adjacent layers, a convergence study was conducted on a percentage difference of the properties. For the purposes of this review, the $\mathrm{E}_{\mathrm{f}}$ variable is used to display the change in properties, however, all properties were changed based on the percentage difference. The highest change in properties was $10 \%$ for a quarter disc model that contained 76 elements $($ seed size $=0.3)$. To improve this, a model that contained 960 elements ( of $50 \%$. Finer meshes were attempted, but did not converge. A 50\% difference was defined as the properties half way between the average of the two layers and the actual layer values. These values were used in this analysis for this thesis, but were not the desired values. 
Table A.1. A 50\% difference from the average value of $\mathrm{E}_{\mathrm{f}}$.

\begin{tabular}{|c|c|c|c|c|}
\hline S & S 50\% & Average & M 50\% & M \\
\hline 320 & 377.5 & 435 & 492.5 & 550 \\
\hline
\end{tabular}

5. Utilizing the 50\%-difference properties, a 6-layered model was created to vary the material properties through the depth of the disc. The top three layers for the S-layer were varied so that the average would amount to the desired S-layer properties. The thought here was that the gradual step between the properties would allow ABAQUS to converge. Unfortunately the model diverged and with only 6 layers, the seed size did not match the 960-element model ( seed size $=0.12)$ used in attempt 4 .

Table A.2. A 6-layered model displaying variations in $\mathrm{E}_{\mathrm{f}}$.

\begin{tabular}{|c|c|c|}
\cline { 2 - 2 } \multicolumn{1}{c|}{} & $\mathbf{E}_{\mathbf{f}}$ & Average \\
\hline S1 & 262.5 & \\
S2 & 320 & 320 \\
\hline S3 & 377.5 & \\
\hline M1 & 492.5 & \\
\hline M2 & 550 & 50 \\
\hline M3 & 607.5 & \\
\hline
\end{tabular}

6. To match a seed size of 0.12, a 10-layer model (960 elements) was created. Similar modeling steps were used to the 6-layer model. The variations in the properties attempted are listed in Table A.3. For variations 1-3, the average values match that of the actual layer properties. In later variations, it was assumed that the S1 layer of variations 1-3 contained properties that 
made the layer too soft and consequently absorbing too much of the strain.

The high strain results in high pressures and causes convergence issues in ABAQUS. For variations 4-6, a majority of the actual S and M layer properties were maintained in the model with the variation only occurring towards the center of the disc.

Table A.3. A 10-layered model displaying variations in $\mathrm{E}_{\mathrm{f}}$.

\begin{tabular}{|c|c|c|c|c|c|c|c|}
\cline { 2 - 4 } \multicolumn{1}{c|}{} & $\mathbf{E}_{\mathbf{f}} \mathbf{1}$ & $\mathbf{E}_{\mathbf{f}} \mathbf{2}$ & $\mathbf{E}_{\mathrm{f}} \mathbf{3}$ & Avg & $\mathbf{E}_{\mathbf{f}} \mathbf{4}$ & $\mathbf{E}_{\mathbf{f}} \mathbf{5}$ & $\mathbf{E}_{\mathbf{f}} \mathbf{6}$ \\
\hline S1 & 262.5 & 285 & 255 & & 320 & 320 & 320 \\
\hline S2 & 320 & 290 & 320 & & 320 & 320 & 320 \\
\hline S3 & 320 & 300 & 320 & \multirow{2}{*}{320} & 320 & 320 & 320 \\
\hline S4 & 320 & 330 & 320 & & 320 & 320 & 377.5 \\
\hline S5 & 377.5 & 395 & 385 & & 377.5 & 435 & 435 \\
\hline M1 & 492.5 & 474 & 485 & & 492.5 & 435 & 435 \\
\hline M2 & 550 & 550 & 550 & & 550 & 550 & 492.5 \\
\hline M3 & 550 & 565 & 550 & \multirow{2}{*}{550} & 550 & 550 & 550 \\
\hline M4 & 550 & 576 & 550 & & 550 & 550 & 550 \\
\hline M5 & 607.5 & 585 & 615 & & 550 & 550 & 550 \\
\hline
\end{tabular}

7. Originally to model the layered analyses, a separate part was created for each layer and all layers were combined using tie constraints. However, developing a one-part model with 6 to 10 layers of properties can produce the same outcome. There was no change in convergence or analysis time, but it was much easier to model the single part.

8. A majority of the analyses utilized a C3D8P element, which is an 8-node brick element. C3D20P elements (20 nodes) were also attempted, but there was no change in convergence and there was a very high increase in computational time. 


\section{A.4 Technical Specifications}

The Biomedical and General Engineering department of Cal Poly provided the computers to run these computational analyses.

Hardware:

Memory: $\quad 11.8 \mathrm{GiB}$

Processor (x8 core): Intel(R) Core(TM) i7 CPU 950 @ 3.07GHz

Operating System:

Fedora: based on Linux

Release 10 (Cambridge)

Kernel Linux 2.6.27.25-170.2.72.fc10.x86_64

GNOME 2.24.3

Software:

ABAQUS/CAE 6.9-2

Build ID: 2009_07_10-10.30.58 92676

(C) Dassault Systemes 2009 


\section{A.5 Statistical Data on COL/GAG Concentrations}

MINITAB

S10+D Inner Core and D0 GAG Concentrations

Two-Sample T-Test and CI

$\begin{array}{ccccc}\text { Sample } & \text { N } & \text { Mean } & \text { StDev } & \text { SE Mean } \\ \text { D0 } & 16 & 4.019 & 0.981 & 0.25 \\ \text { S10+D In } & 5 & 5.493 & 0.307 & 0.14\end{array}$

Difference $=\mathrm{mu}(1)-\mathrm{mu}(2)$

Estimate for difference: -1.474

95\% upper bound for difference: -0.987

T-Test of difference $=0($ vs $<):$ T-Value $=-5.24 \mathrm{P}$-Value $=0.000 \mathrm{DF}=18$

S10+D Outer Ring and D0 GAG Concentrations

Two-Sample T-Test and CI

$\begin{array}{ccccc}\text { Sample } & \text { N } & \text { Mean } & \text { StDev } & \text { SE Mean } \\ \text { D0 } & 16 & 4.019 & 0.981 & 0.25 \\ \text { S10+D Out } & 5 & 12.99 & 1.74 & 0.78\end{array}$

Difference $=\mathrm{mu}(1)-\mathrm{mu}(2)$

Estimate for difference: -8.968 
95\% upper bound for difference: -7.230

T-Test of difference $=0(\mathrm{vs}<): \mathrm{T}-$ Value $=-11.00 \mathrm{P}-$ Value $=0.000 \mathrm{DF}=4$

S10+D Inner Core and D0 COL Concentrations

Two-Sample T-Test and CI

$\begin{array}{ccccc}\text { Sample } & \text { N } & \text { Mean } & \text { StDev } & \text { SE Mean } \\ \text { D0 } & 16 & 6.34 & 1.73 & 0.43 \\ \text { S10+D In } & 5 & 5.34 & 1.13 & 0.51\end{array}$

Difference $=m u(1)-m u(2)$

Estimate for difference: 1.003

95\% CI for difference: $(-0.481,2.487)$

$\mathrm{T}$-Test of difference $=0($ vs not $=): \mathrm{T}-$ Value $=1.51 \mathrm{P}-$ Value $=0.163 \mathrm{DF}=10$

S10+D Outer Ring and D0 GAG Concentrations

Two-Sample T-Test and CI

$\begin{array}{ccccc}\text { Sample } & \text { N } & \text { Mean } & \text { StDev } & \text { SE Mean } \\ \text { D0 } & 16 & 6.34 & 1.73 & 0.43 \\ \text { S10+D Out } & 5 & 5.152 & 0.693 & 0.31\end{array}$


Difference $=\mathrm{mu}(1)-\mathrm{mu}(2)$

Estimate for difference: 1.190

95\% lower bound for difference: 0.265

T-Test of difference $=0($ vs $>):$ T-Value $=2.24 \mathrm{P}-$ Value $=0.019 \mathrm{DF}=17$

S10+D Inner Core and Outer Ring COL Concentrations

Two-Sample T-Test and CI

$\begin{array}{lcccc}\text { Sample } & \text { N } & \text { Mean } & \text { StDev } & \text { SE Mean } \\ \text { Inner } & 5 & 5.34 & 1.13 & 0.51 \\ \text { Outer } & 5 & 5.152 & 0.693 & 0.31\end{array}$

Difference $=\mathrm{mu}(1)-\mathrm{mu}(2)$

Estimate for difference: 0.187

95\% CI for difference: $(-1.266,1.640)$

$\mathrm{T}$-Test of difference $=0($ vs not $=): \mathrm{T}-$ Value $=0.31 \mathrm{P}-$ Value $=0.764 \mathrm{DF}=6$

S10+D and FSB COL Concentrations

Two-Sample T-Test and CI

$\begin{array}{ccccc}\text { Sample } & \text { N } & \text { Mean } & \text { StDev } & \text { SE Mean } \\ \text { FSB } & 6 & 4.78 & 3.36 & 1.4 \\ \text { S10+D } & 5 & 5.196 & 0.797 & 0.36\end{array}$

Difference $=\mathrm{mu}(1)-\mathrm{mu}(2)$

Estimate for difference: -0.42 
95\% upper bound for difference: 2.44

T-Test of difference $=0($ vs $<): T-$ Value $=-0.29 \mathrm{P}-$ Value $=0.391 \mathrm{DF}=5$

S10+D Inner Core and Outer Ring GAG Concentrations

Two-Sample T-Test and CI

$\begin{array}{ccccc}\text { Sample } & \text { N } & \text { Mean } & \text { StDev } & \text { SE Mean } \\ \text { Inner } & 5 & 5.493 & 0.307 & 0.14 \\ \text { Outer } & 5 & 12.99 & 1.74 & 0.78\end{array}$

Difference $=m u(1)-m u(2)$

Estimate for difference: -7.494

95\% upper bound for difference: -5.810

T-Test of difference $=0($ vs $<):$ T-Value $=-9.49 \mathrm{P}-$ Value $=0.000 \mathrm{DF}=4$

S10+D and FSB Inner Core GAG Concentrations

Two-Sample T-Test and CI

$\begin{array}{ccccc}\text { Sample } & \text { N } & \text { Mean } & \text { StDev } & \text { SE Mean } \\ \text { FSB } & 6 & 4.60 & 1.88 & 0.77 \\ \text { S10+D } & 5 & 5.493 & 0.307 & 0.14\end{array}$

Difference $=\mathrm{mu}(1)-\mathrm{mu}(2)$

Estimate for difference: -0.892

95\% upper bound for difference: 0.676 
T-Test of difference $=0($ vs $<):$ T-Value $=-1.15$ P-Value $=0.152$ DF $=5$

S10+D and FSB Outer Ring GAG Concentrations

Two-Sample T-Test and CI

$\begin{array}{ccccc}\text { Sample } & \text { N } & \text { Mean } & \text { StDev } & \text { SE Mean } \\ \text { FSB } & 6 & 6.41 & 3.16 & 1.3 \\ \text { S10+D } & 5 & 12.99 & 1.74 & 0.78\end{array}$

Difference $=\mathrm{mu}(1)-\mathrm{mu}(2)$

Estimate for difference: -6.58

95\% upper bound for difference: -3.73

T-Test of difference $=0($ vs $<):$ T-Value $=-4.37$ P-Value $=0.002$ DF $=7$ 